Novel evaluation method of acoustic emission data based on statistical fiber bundle cells

Vas L. M., Kocsis Z., Czigány T., Tamás P., Romhány G.

Accepted for publication in Journal of Composite Materials

Published in 2019

DOI: $10.1177 / 0021998319826666$ 


\title{
NOVEL EVALUATION METHOD OF ACOUSTIC EMISSION DATA BASED ON STATISTICAL FIBER BUNDLE CELLS
}

\author{
${ }^{1}$ László Mihály VAS $*{ }^{*}$ Zoltán KOCSIS, ${ }^{1,3}$ Tibor CZIGÁNY, ${ }^{4}$ Péter TAMÁS, \\ ${ }^{1}$ Gábor ROMHÁNY \\ ${ }^{1}$ Department of Polymer Engineering, Faculty of Mechanical Engineering, Budapest \\ University of Technology and Economics, Müegyetem rkp. 3., H-1111 Budapest, Hungary \\ ${ }^{2}$ CADFEM GmbH, Wagenseilgasse 14, A-1120 Wien, Austria \\ ${ }^{3}$ MTA-BME Research Group for Composite Science and Technology, Müegyetem rkp. 3., H- \\ 1111 Budapest, Hungary \\ ${ }^{4}$ Department of Mechatronics, Optics and Mechanical Engineering Informatics, Faculty of \\ Mechanical Engineering, Budapest University of Technology and Economics, \\ Müegyetem rkp. 3., H-1111 Budapest, Hungary \\ *corresponding author: vas@pt.bme.hu
}

\begin{abstract}
In general, the aim of acoustic emission (AE) assisted tensile tests of composite materials is to identify and characterize the damage and failure modes of the specimens. This paper presents a fiber-bundle-cells (FBC) based statistical model, which provides a possible solution to the problem of characterizing the mechanical and failure behavior of the material. The model, based on the results of mechanical tests and $\mathrm{AE}$ measurements, decomposes the measured $\mathrm{AE}$ event number and tensile force-load time processes into components corresponding to the different damage modes. The AE events belonging to different failure modes are described by inhomogeneous Poisson point processes, while failures are modeled with the breakage of fibers as elementary parts of the sample. Hence damage modes can be characterized with the number fraction, and the tensile strength and signal energy distributions of the components. Moreover, the variation of the number fraction of the intact or damaged fibers as a function of the load time can be calculated and depicted as well. As reliability function or a kind of damage map, it reveals the mechanical load-bearing ability of the material tested.

The applicability of the model is demonstrated by compact tension testing and the comparison of short and long glass fiber reinforced VERTON PP sheets and injection molded wood fiber reinforced PP composites.
\end{abstract}

Keywords: non-destructive evaluation, AE measurement, fiber bundle model, Poisson event process, failure mode characterization, reliability, damage map, polymer-matrix composites

\section{Introduction}

Nowadays one of the most effective non-destructive structural-mechanical tests is the detection and analysis of acoustic emission (AE) signals in materials subjected to mechanical load. The most important objectives of utilizing AE data are to identify, classify, and 
characterize the different damage or failure modes that can occur e.g. in inhomogeneous materials, such as fiber-reinforced composites and to qualify the fracture mechanical behavior of materials or the structural-mechanical state of machine parts, or health monitoring, as it is usually called..$^{1-4}$

The AE signal generated by an energy release which is induced by a sudden irreversible local change in mechanically loaded material is a burst type (Figure 1) vibration of 30-600 kHz. The local change is usually crazing or micro-cracking. The vibration can be detected with suitable sensors and converted into an electric signal. ${ }^{1-4}$ The AE signal may be a continuous and/or long damping vibration but its source is usually an external noise. On the other hand, acoustic emission of lower frequency $(0,1-1,0 \mathrm{kHz})$ can also be generated by e.g. the blade tip of axial fans. ${ }^{5,6}$ Similar vibrations can be observed in vehicle parts such as airplane wings or a fuselage. ${ }^{1-4}$

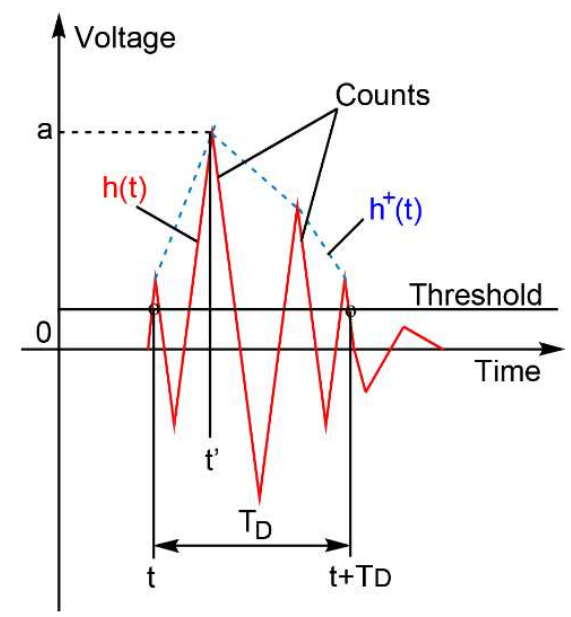

Figure 1. Numerical descriptors of a burst type AE signal in the time domain

\section{AE signal processing}

AE signals are detected as a function of time when they exceed a given threshold level (Figure 1), hence the time domain characteristics of single AE signals $(h)$ are fundamental. Such characteristics are the numerical properties of the waveform, e.g. detection time $(t)$, which is the time of the first threshold crossing, maximum amplitude $(a)$ and the time belonging to that $\left(t^{\prime}\right)$, rise time $\left(T_{R}=t^{\prime}-t\right)$, duration time $\left(T_{D}\right)$, attenuation time $\left(T_{D}-T_{R}\right)$, counts $(C)$, which is the number of positive crossings, and signal energy $\left(U^{+}\right)$, which is the measure of the area under the upper envelope curve $\left(h^{+}\right)$of the signal (MARSE) or as the mean square value of the signal. ${ }^{2}{ }^{4}$ Based on the data of single AE signals, various statistics can be calculated and plotted, such as the number of hits or cumulative energy versus time, amplitude or energy histograms, or counts versus the amplitude cross-plot. ${ }^{2,4}$

To detect the different failure modes (crazing, micro-cracking in matrix, debonding between fiber and matrix, pullout or break of fiber, micro-buckling, delamination, propagation of macro-crack) in different polymer materials, the simplest and conventional method is to analyze changes in the load-deformation relationship obtained by mechanical test and the time domain $\mathrm{AE}$ data such as the number of $\mathrm{AE}$ events and the maximum amplitude versus time plot and to compare them to each other and to the amplitude histogram and possibly other descriptive statistics. ${ }^{7-20}$ 
With the help of fast Fourier transformation (FFT), the frequency components of the single AE signals can be characterized by the amplitude or power spectrum in the frequency domain. Their description parameters are the bandwidth $(B)$, the mean frequency $\left(f_{m}\right)$, the frequency of the maximum amplitude component $\left(f_{P}\right)$, and the amplitude $\left(A_{P}\right)$ or energy $\left(U_{P}\right)$ of the maximum peak. Similar parameters can be obtained from the mean spectra of a series of AE signals, and histograms and/or cross-plots can be calculated from the data of single AE signals. ${ }^{2,4}$

Special techniques can provide information in both the time and the frequency domain, resulting in time-dependent spectrum statistics. ${ }^{2,4}$ The classic method is the short time FFT (SFFT) technique, which uses a moving time window. In the case of wavelet transformations (WT), AE signals are analyzed with the help of various wavelet components.

The signal transformations mentioned above provide additional or in itself usable information about the sources and failure modes in the frequency domain ${ }^{21-26}$ or both in the time and the frequency domains ${ }^{27-36}$. In the latter case, wavelet transformations based on $\mathrm{Haar}^{28}$ or Gabor $^{27}$, ${ }^{33}$ wavelet components as well as Hilbert-Huang transform ${ }^{30}$ can be used to decompose the AE signals and calculate the energy of the components. ${ }^{27-36}$ In the case of thin plates, modal analysis can be applied to study and separate extensional and flexural Lamb waves. ${ }^{37,}{ }^{38}$ The finite element method (FEM) can give a good base for wave discrimination. ${ }^{38}$

The localization of a single AE signal in the specimen tested provides information about the sources in the space domain. It can be performed by applying several sensors and making use of the difference between the detection times of single AE hits and the speed of sound in the material tested. ${ }^{2,4}$ Hafizi et al. ${ }^{33}$ proposed a signal attenuation-based source mapping instead of sound velocity mapping. From these data, spatial histograms, the damage zone and its development or crack initiation and propagation can be determined and monitored. ${ }^{31,39-41}$ The sentry function, which is the logarithm of the ratio of the strain energy (determined from the load-displacement plot) and the energy of the AE signal can be applied to study the initiation and growth of the delamination process. ${ }^{17,} 24$ Artificial neural network (ANN) based evaluation could be more effective for source localization ${ }^{40}$ or predicting crack length ${ }^{41}$.

Another possibility to locate damages and characterize their geometry is provided by the ultrasonic non-destructive testing method using the analysis of the damage induced anisotropy based on measuring quasi-static indentation ${ }^{42}$ or shear wave birefringence ${ }^{43}$. The accuracy of measuring damage size has also been analyzed using different ultrasonic testing modes. ${ }^{44}$

\section{Identification, classification and characterization of micro-failure modes}

The conventional method for identifying, classifying and characterizing damage modes is to employ descriptors of the AE signal in the time or the frequency domain. The comparison of the variation of mechanical stress, cumulative $\mathrm{AE}$ event count, and $\mathrm{AE}$ signal amplitudes or energies as a function of the strain or time gives the sequence of dominant amplitudes. With information about the sequence of the micro-failure modes, they can be matched to the dominant amplitude intervals, and the results can be validated by scanning electron microscopy (SEM), which can prove at least the existence of the assumed damage modes ${ }^{8,9}$, $12,18,24,26,29,32$ as well as light microscopy ${ }^{9}, 11,22,39$, X-ray photographs ${ }^{7}$, and infrared 
thermography ${ }^{39}$. The latter can provide data about the place and nature of the observed damage as well.

Zhuang and Yan ${ }^{15}$ identified five damage modes in self-reinforced PE composite laminates with a special layer order and related them to dominant amplitude intervals of detected $\mathrm{AE}$ signals. It should be noted that the amplitude ranges of matrix cracking and debonding as well as fiber pull-out and delamination strongly overlapped.

A more correct identification of damage modes can be based on completing AE data with special micromechanical tests, such as pure matrix, single-fiber ${ }^{23,27}$ or fiber bundle tensile tests ${ }^{9,13,16,35}$ in order to investigate the fiber pull-out and breakage in single and/or multi-fiber composite specimens ${ }^{22,23}$. Using such special specimens made from carbon fibers and an epoxy matrix, $\mathrm{Ni}$ and Iwamoto $^{27}$ found that the peak frequencies of three failure modes such as matrix cracking, debonding and fiber break fell into separate ranges. On the other hand, testing carbon/epoxy composites, de Groot et al. ${ }^{21}$ concluded that on the basis of AE signal frequency bands, matrix cracking and fiber breakage can be separated, although in the case of debonding and fiber pull out, the frequency bands turned out to be interchanged. In addition, Lomov et al. ${ }^{25}$ reported that peak frequencies did not correlate with the sequence of observed damage modes, therefore they proposed to use the AE signal energy as the main parameter for distinction.

More advanced methods of the discrimination and classification of single AE signals are based on multivariate statistical analysis, including intelligent pattern recognition techniques. ${ }^{45-54}$ Clustering techniques (CT) such as hierarchical ${ }^{51,54}$, unsupervised k-means ${ }^{47}$, ${ }^{49-51}$ or fuzzy C-means ${ }^{51,52}$ algorithms and unsupervised waveform clustering ${ }^{53}$ are suitable tools for filtering noise ${ }^{49}$ and classifying the AE signals based on their numerical descriptors as variables. In order to reduce the number of variables, which can be more than 10-20, principal component analysis (PCA) ${ }^{45,50-52}$ and Sammon mapping ${ }^{45}$ can be used to create new (possibly uncorrelated) variables without losing significant information. Hierarchical clustering does not require fixing the number of clusters but it needs large storage capacity while unsupervised methods use iteration, therefore in general the number of clusters need not be known. In the latter case, the result of clustering strongly depends on the initial random choice of clusters. Harmony search is a novel meta-heuristic algorithm that can be applied to find proper or possibly optimal centers for clustering, among others ${ }^{47,48}$. The artificial neural network (ANN) technique is also suitable for pattern recognition, that is, classifying $\mathrm{AE}$ signals. $^{46}$

Considering the mechanical nature of damage, the characterization of the identified and classified failure modes and their onset should be based on statistical descriptors of strength properties corresponding to the loading mode applied, such as quasi-static tensile or bending strength, fatigue or fracture mechanical data. Some researchers applied Weibull distribution to describe the strength of single fibers as constituents of the fibrous structures tested. ${ }^{9}, 16,25$ Bocchieri et al. ${ }^{37}$ related the normalized cumulative number of AE events vs. axial stress to the empirical strength distribution function of micro-cracks. Fiber bundle test and AE signal analysis were used to characterize single fiber break events. ${ }^{13}, 16,35$ Hill and Okoroafor ${ }^{9}$ employed a classical linear fiber bundle model for describing and analyzing fiber bundle tests and $\mathrm{AE}$ results based on the distribution function of single Kevlar and glass fibers. 
The results of AE signal processing and identification, classification and characterization of micro-failure modes can be applied to the mechanical dimensioning of parts or constructions made of fiber-reinforced materials and also to their structural health monitoring. ${ }^{4,19,30,36}$

Despite all the results discussed above, there is a need for an effective deducing procedure that is based on reliable relations between the AE signals and failure modes, which 'is still one of the major challenges' in this field, according to Al-Jumaili et al. (2014). ${ }^{51}$

\section{Objectives}

This paper presents a statistical fiber-bundle-cells ${ }^{55-61}$ based novel modeling and evaluating method for classifying and characterizing $\mathrm{AE}$ signals, which can manage overlaps in histograms or spectrums by the simultaneous decomposition of the stress-strain curve, the cumulative number of $\mathrm{AE}$ events and any cumulative statistics of $\mathrm{AE}$ descriptors. The fitted FBC model makes it possible to analyze the mechanical reliability of the material and construct a kind of damage map for characterizing the failure behavior. We demonstrate the applicability of this method by evaluating some tensile tests and AE measurements.

\section{Theoretical Considerations}

\subsection{Statistical fiber-bundle-cells (FBCs)}

The FBC modeling method is based on some idealized statistical fiber bundles called fiberbundle-cells (FBCs) which can be used as building elements of a model network created by parallel and/or serial connections. ${ }^{55-59}$ Idealized fiber bundles are defined as fiber classes containing fibers of the same geometrical (shape, disposition) and mechanical properties (strain state, gripping by the surroundings) (Figure 2). In the simplest case, the fibers are ideally elastic (E) with a linear or non-linear relationship between the strain and load, although they break at a random strain value.

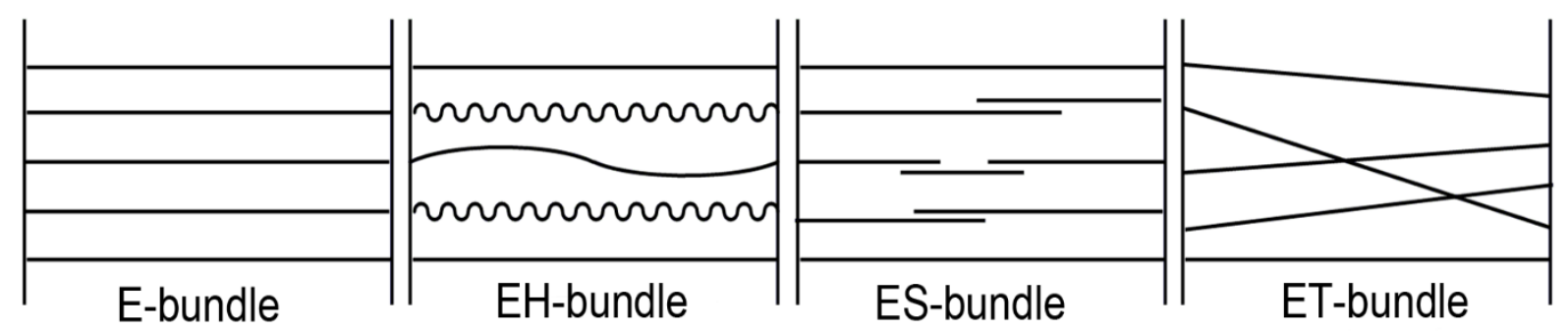

Figure 2. Structural scheme of idealized fiber bundle cells ${ }^{58}$

The fibers of an E-bundle are straight and parallel to the direction of the tensile load without any pretension and they are ideally gripped, that is, they do not slip out of the grips. Additional fiber classes can be created if one or more certain ideal properties are lacking. The fibers may be loose or pre-stressed in the EH-bundle and they are oblique in the ET-bundle. In the ES-bundle the fibers can slip out of the grips or they can create fiber chains with slipping 
bonds and their damage mode may be breakage or slippage. These properties are combined in the EHS, EHT, EST, and EHST bundles. In general, the shape, position, and strength parameters of fibers are assumed to be independent stochastic variables.

Their weighted parallel connection can provide an FBC model for describing the mechanical behavior of a fibrous sample during a tensile test of constant rate elongation including both the deformation and the damage processes up to the last fiber breakage.

In this paper a simplified FBC modeling method is used, where E-bundles with a nonlinear tensile characteristic are applied to modeling and analyzing the tensile and AE behavior of polymer composite samples. The damage mode of an E-bundle is the breakage of the single fibers. The tensile force of the E-bundle (Figure 2) as a function of bundle strain creates a stochastic process and its expected value (denoted by $E$ ) can be calculated with the following formula $^{58,59}$ :

$$
E[F(u ; c, b)]=N \cdot \kappa_{I}(u ; c)\left(1-Q_{u B}(u ; b)\right)=\kappa(u ; c)\left(1-Q_{u B}(u ; b)\right)=\kappa(u ; c) R(u ; b)(1)
$$

where $F \geq 0$ and $u \geq 0$ are the bundle force and elongation (or strain), respectively, $N$ is the number of fibers, $Q_{u B}$ is the distribution function of the breaking elongation $\left(u_{B}\right)$ of the fibers, while $\kappa_{I}(u), \kappa_{I}(0)=0$, is the tensile characteristic of the single fibers, which is linear in simple cases, while $\kappa(u)=N \kappa_{I}(u)$ is the tensile characteristic of the fiber bundle and parameter vectors $c$ and $b$ denote the parameters of $\kappa(u)$ and $Q_{u B}(u)$, respectively. In Equation (1), the tensile characteristic, $\kappa(u)$, describes the failureless behavior of the fibrous structure while $\mathrm{R}(\mathrm{u})=1$ $Q_{u B}(u)$ gives the fraction of the intact fibers at the given load level, hence it is a kind of reliability function and represents the statistical properties of the damage process.

\subsection{FBC based modeling of the AE signal process}

Consider a fiber-reinforced composite material the matrix of which may also contain fillers. A tensile test is performed on some samples of this material with gauge length $l_{0}$ under constant rate elongation $\left(\dot{u}_{0}\right)$, consequently the time $(t)$ and the elongation $(u)$ or strain $(\varepsilon)$ are proportional to each other:

$$
\begin{aligned}
& u=\Delta l=l-l_{0}=\dot{u}_{0} t \\
& \varepsilon=\frac{\Delta l}{l_{0}}=\frac{\dot{u}_{0}}{l_{0}} t=\dot{\varepsilon}_{0} t
\end{aligned}
$$

where $l$ is the deformed length of the sample and the strain rate, $\dot{\varepsilon}_{0}$, is defined by Equation (3). The tensile force $(F)$ is recorded as a function of the time or elongation.

\section{Flaw populations and properties of AE signals}

Let us assume that there are different flaw populations of $1 \leq r<\infty$ types in the material sample before tensile testing, which are potential AE sources. They represent elementary failure modes, such as matrix micro-cracks, filler-matrix or fiber-matrix debonding, fiber pull-out, and fiber breakage that can occur in a possible filled and fiber-reinforced polymer material. 
Let $p_{\mathrm{i}}\left(i=1, \ldots, r ; p_{1}+\ldots+p_{\mathrm{r}}=1\right)$ be the probability that an arbitrary flaw belongs to the $i^{\text {th }}$ type population related to the $i^{\text {th }}$ (elementary) failure mode.

The $j^{\text {th }}$ AE signals generated by a $i^{\text {th }}$ type flaw during a tensile test and detected as a voltagetime function (Figure 1) can be described in the following mathematical form $(0 \leq t)$ :

$$
h_{i j}(t)=\propto_{i j} g_{i}\left(t-\tau_{i j}\right)
$$

where $\alpha_{i j}>0$ and $\tau_{i j}>0$ are the maximum amplitude and the detection (arrival) time, respectively. They are independent stochastic variables, and $-1 \leq g_{i}(t) \leq 1$ is the signal shape function of unit maximum amplitude belonging to the $i^{\text {th }}$ type flaw and it may be confounded by additive random noise. The value of $h_{i j}(t)$ is the threshold level if $t=\tau_{i j}$ or $t=\tau_{i j}+\tau_{D i j}$. The distribution functions of detection time and amplitude are $Q_{\tau_{i}}$ and $Q_{\alpha i}$, respectively, and assumed to be dependent on the failure mode $(i=1, \ldots, r)$ only.

The peak time $\tau_{i j}^{\prime}$ belongs to the maximum amplitude and its relation to the detection (arrival) time, $\tau_{\mathrm{ij}}$, and the rise time, $\tau_{R i j}$, is given by (Figure 1 ):

$$
\tau_{i j}^{\prime}=\tau_{i j}+\tau_{R i j}: \quad h_{i j}\left(\tau_{i j}^{\prime}\right)=\propto_{i j} g_{i}\left(\tau_{R i j}\right)=\propto_{i j}, \quad g_{i}\left(\tau_{R i j}\right)=1
$$

where all the AE signal times introduced above are stochastic variables.

The AE signal, $h_{i j}(\mathrm{t})$, is a decaying vibration of burst type with finite duration time. In the time domain, the whole resultant stochastic AE process can be given as the sum of the single signals:

$$
h(t)=\sum_{i j} h_{i j}(t)=\sum_{i=1}^{r} \sum_{j} \propto_{i j} g_{i}\left(t-\tau_{i j}\right)
$$

Consequently, the detected voltage-time process is a realization of $h(t)$. Obviously, two signals in this process may overlap each other hence their sum can be detected as a single one with amplified or reduced amplitude compared to that of the addends.

The signal energy $\left(U_{i j}^{+}\right)$is defined in Figure 1 (MARSE) as the integral of the signal envelope curve, $h_{i j}^{+}$, which is an upper estimation for the integral of the signal absolute value $\left(U_{i j}\right)$ :

$$
U_{i j}^{+}=\int_{\tau_{i j}}^{\tau_{i j}+\tau_{D i j}} h_{i j}^{+}(t) d t \geq U_{i j}=\int_{0}^{\infty}\left|h_{i j}(t)\right| d t=\propto_{i j} \int_{\tau_{i j}}^{\tau_{i j}+\tau_{D i j}}\left|g_{i j}\left(t-\tau_{i j}\right)\right| d t
$$

The quantity $U_{i j}$ is proportional $(\sim)$ to the mechanical energy if voltage $\left(h_{i j}\right) \sim$ stress is true since the time $\sim$ deformation relation stands. In signal analysis, the signal energy is usually characterized by the integral of the squared signal, which is proportional to the electric energy $\left(U_{E i j}\right)$ dissipated on a conductive resistance: ${ }^{62}$

$$
U_{E i j}=\int_{0}^{\infty} h_{i j}^{2}(t) d t=\propto_{i j}^{2} \int_{\tau_{i j}}^{\tau_{i j}+\tau_{D i j}} g_{i j}^{2}\left(t-\tau_{i j}\right) d t
$$

The cumulative AE signal energy in interval $(0, t)$ is characterized by the integral of the squared amplitude process: 


$$
U_{E}(t) \rightarrow U_{A}(t)=\int_{0}^{t} A^{2}(v) d v=\sum_{i=1}^{r} \sum_{j} \propto_{i j}^{2} \theta\left(t-\tau_{i j}\right)
$$

where $U_{A}(t)$ can be regarded as a kind of signal power and $\theta(t)$ is the unit step function introduced by Heaviside ${ }^{62}$.

\section{Elementary lifetime and the event number process}

Detection time, $\tau_{i j}$, can be regarded as the lifetime of the volume element that is the source of the given AE signal. The detection time values create a stochastic point or event process on the time axis, where the number of AE events in interval $(0, t)$ denoted by $v(t)$ is the sum of its components, $v_{i}(t)(\mathrm{i}=1, \ldots, r)$, corresponding to the failure modes and this process can be expressed as a cumulative step function determined by the detection times $\left(\tau_{i j}\right)$

$$
v(t)=\sum_{i=1}^{r} v_{i}(t)=\sum_{i=1}^{r} \sum_{j} \theta\left(t-\tau_{i j}\right)
$$

Denoting the observed realization of the number events $v(t)$ and $v_{i}(t)(i=1, \ldots, r)$ by $n(t)$ and $n_{i}(t)$ respectively we get a similar sum:

$$
n(t)=\sum_{i=1}^{r} n_{i}(t)=\sum_{i=1}^{r} \sum_{j} \theta\left(t-t_{i j}\right)
$$

where $t_{i j}$ is the observed value of $\tau_{i j}$. The expected value of the process, $v(t)$, is a sum as well, which can be approximated with the mean $(\bar{n})$ of some $(\geq 1)$ observed realizations:

$$
\Lambda(t)=E(v(t))=\sum_{i=1}^{r} \Lambda_{i}(t)=\sum_{i=1}^{r} E\left(v_{i}(t)\right) \approx \bar{n}(t)=\sum_{i=1}^{r} \bar{n}_{i}(t)
$$

According to experiences ${ }^{7-21,60}$, the mean of this point or event/hit process, $\Lambda(t)$, changes with increasing strain, which is proportional to the time in the case of a tensile test. In general, in most cases these points signify events, which can be treated as an inhomogeneous Poisson process: ${ }^{.58,63}$

$$
P(v(t)=n)=\frac{\Lambda^{n}(t)}{n !} e^{-\Lambda(t)}
$$

where the parameter, $\Lambda(t)$, equals the expected value of $v(t)$ and is the integral of the mean point density $\lambda(t)$ :

$$
\Lambda(t)=E(v(t))=\int_{0}^{t} \lambda(u) d u=\sum_{i=1}^{r} \int_{0}^{t} \lambda_{i}(u) d u \Rightarrow \lambda(t)=\sum_{i=1}^{r} \lambda_{i}(t)
$$

where $\lambda_{i}(t)$ is the point density of $v_{i}(t)$. In the case of homogenous processes, all the point densities are constant:

$$
\Lambda(t)=\lambda t=\sum_{i=1}^{r} \lambda_{i} t \Rightarrow \lambda=\sum_{i=1}^{r} \lambda_{i}
$$


although in general, this does not stand for tensile test measurements of polymers.

\section{Strength characteristics of the failure modes and the event number process}

Let $N$ be the expected number of critical flaws that fail independently of each other, causing the ultimate failure of the sample during the tensile test with the given setting parameters and assuming a long enough observational time. In this case, let $N_{i}$ be the number of critical flaws in the $i^{\text {th }}$ population $(i=1, \ldots, r)$, which is given by:

$$
N_{i}=p_{i} N
$$

Supposing that each observed AE signal can be evaluated in every respect within the total observation time, $T$, yields:

$$
\mathrm{T} \geq \max _{i j}\left(t_{i j}+T_{D i j}\right)
$$

where $t_{i j}$ and $T_{D i j}$ are the detection and duration times of the observed AE signal respectively and the right side is a realization of the lifetime of the sample. Accordingly, the total number $(N)$ of the detectable AE signals can be approximated with the observed mean number of AE events, obtained by averaging some $(\geq 1)$ measurements:

$$
N=\sum_{i=1}^{r} N_{i} \approx \bar{n}(T)=\sum_{i=1}^{r} \bar{n}_{i}(T)
$$

consequently, the probability that an arbitrary critical flaw is of the $i^{\text {th }}$ type can be estimated with the help of their ratio:

$$
p_{i}=\frac{N_{i}}{N}=\lim _{t \rightarrow \infty} \frac{\Lambda_{i}(t)}{\Lambda(t)} \approx \frac{\Lambda_{i}(T)}{\Lambda(T)} \approx \frac{\bar{n}_{i}(T)}{\bar{n}(T)}=\hat{p}_{i} ; \quad \sum_{i=1}^{r} \hat{p}_{i}=1
$$

On the basis of this, it can be supposed that the sample is built up of $N$ elements, each of which contains a single critical flaw. Ultimate failure occurs when the last still intact element fails determining the lifetime $(\tau)$ of the sample subjected to the tensile load. Hence the lifetime of the sample is determined by the element lifetimes, which can be identified with the detection times. Their distribution depends on the failure mode. Thus the distribution function of the element lifetime, $Q_{i i}(t)$, determines the distribution function of a kind of breaking strain $\left(\varepsilon_{B}\right)$ providing the strength characteristics of the $i^{\text {th }}$ failure mode:

$$
Q_{\tau i}(t)=P\left(\tau_{i j}<t\right)=P\left(\varepsilon_{B i j}=\dot{\varepsilon}_{0} \tau_{i j}<\dot{\varepsilon}_{0} t=\varepsilon\right)=Q_{\varepsilon B i}(\varepsilon)
$$

On the other hand, Equation (20) gives what fraction of the volume elements containing the $i^{\text {th }}$ type critical flaws failed in the interval $(0, t)$ :

$$
Q_{\tau i}(t)=P\left(\tau_{i j}<t\right) \approx \frac{\bar{n}_{i}(t)}{\bar{n}_{i}(T)}=\frac{\#\left\{j: \tau_{i j}<t\right\}}{\#\left\{j: \tau_{i j}<T\right\}}=\widehat{Q}_{\tau i}(t)
$$


where \# denotes the number of set elements and $\hat{Q}_{\tau i}$ is the statistical estimation of $Q_{\tau i}$. Hence the expected value of the number of events can be obtained with:

$$
\begin{gathered}
\Lambda_{i}(t)=E\left(v_{i}(t)\right)=N_{i} Q_{\tau i}(t)=N_{i} \int_{0}^{t} q_{\tau i}(z) d z \approx \Lambda_{i}(T) Q_{\tau i}(t) \approx \bar{n}_{i}(t)=\bar{n}_{i}(T) \hat{Q}_{\tau i}(t)= \\
\bar{n}_{i}(T) \int_{0}^{t} \hat{q}_{\tau i}(z) d z
\end{gathered}
$$

assuming that the density functions, $q_{\tau i}$, exist. Comparing Equations (14) and (22) yields:

$$
\lambda_{i}(t)=\mathrm{N}_{i} q_{\tau i}(t) \approx \Lambda_{i}(T) q_{\tau i}(t) \approx \hat{\lambda}_{i}(t)=\bar{n}_{i}(T) \hat{q}_{\tau i}(t)
$$

From the results above, we get:

$$
\begin{gathered}
\Lambda(t)=E(v(t))=\sum_{i=1}^{r} \mathrm{~N}_{i} Q_{\tau i}(t)=\mathrm{N} \sum_{i=1}^{r} p_{i} Q_{\tau i}(t) \approx \Lambda(T) \sum_{i=1}^{r} p_{i} Q_{\tau i}(t) \approx \bar{n}(t)= \\
\sum_{i=1}^{r} \bar{n}_{i}(T) \hat{Q}_{\tau i}(t)=\bar{n}(T) \sum_{i=1}^{r} \hat{p}_{i} \hat{Q}_{\tau i}(t)
\end{gathered}
$$

\section{Signal amplitude and energy statistics}

Hence, the expected value of the number of AE events is proportional to the weighted sum of the lifetime distribution functions related to the different failure modes. Similarly, the resultant distribution of the signal maximum amplitudes, $\alpha_{i j}$, can be calculated as a weighted sum defining a mixture distribution (Figure 3; $a$ and $t$ are the observed values of $\alpha_{i j}$ and $\tau_{i j}$ respectively):

$$
\mathrm{Q}_{\propto}(\mathrm{x})=P(\propto<x)=\sum_{i=1}^{r} p_{i} P\left(\propto_{i}<x\right)=\sum_{i=1}^{r} p_{i} Q_{\propto i}(x)=\sum_{i=1}^{r} \int_{0}^{x} p_{i} q_{\propto i}(z) d z
$$

supposing that the density functions, $q_{\alpha i}$, exist.

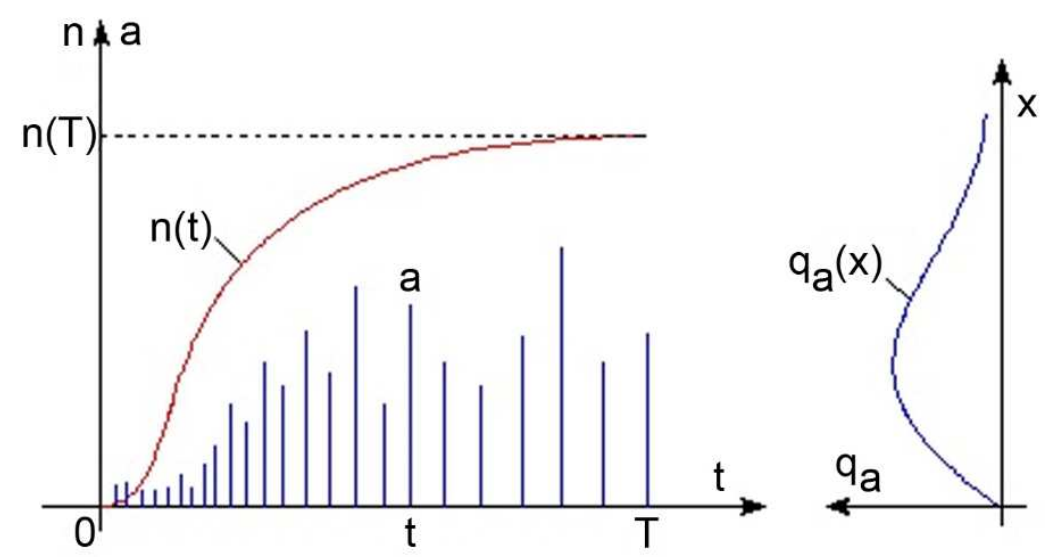

Figure 3. Schematic of usual AE measurement results $\left(q_{a}=\hat{q}_{\propto}\right)$

According to Equation (9), the distribution of energy determined by the squared maximum amplitudes can be calculated from that of the amplitude: 


$$
\mathrm{Q}_{U E}(\mathrm{z})=P\left(\propto^{2}<z\right)=\sum_{i=1}^{r} p_{i} P\left(\propto_{i}^{2}<z\right)=\sum_{i=1}^{r} p_{i} Q_{\propto i}(\sqrt{z})=\sum_{i=1}^{r} \int_{0}^{\sqrt{z}} p_{i} q_{\propto i}(x) d x
$$

\section{The FBC model of deformation and the damage process}

Besides the detected AE signals and the event number process, the recorded stress-strain curve has also got a lot of information about not only deformation behavior but the damage process during the tensile test as well. On the basis of the relations discussed above, it can be supposed that the stress-strain curve can be decomposed into components corresponding to the failure modes.

It was assumed above that the sample is built up of elements containing critical flaws that fail during the tensile test. The number of such elements is $N$ and the ultimate failure of the sample occurs when the last intact element fails. These elements can be regarded as fibers and the interruption of their tensile force transfer caused by fiber break can model the elementary failure that generates an AE signal. Disregarding the last one, an elementary failure does not cause the total failure of the sample, therefore these model-fibers are parallel connected, creating a fiber bundle (Figure 4).

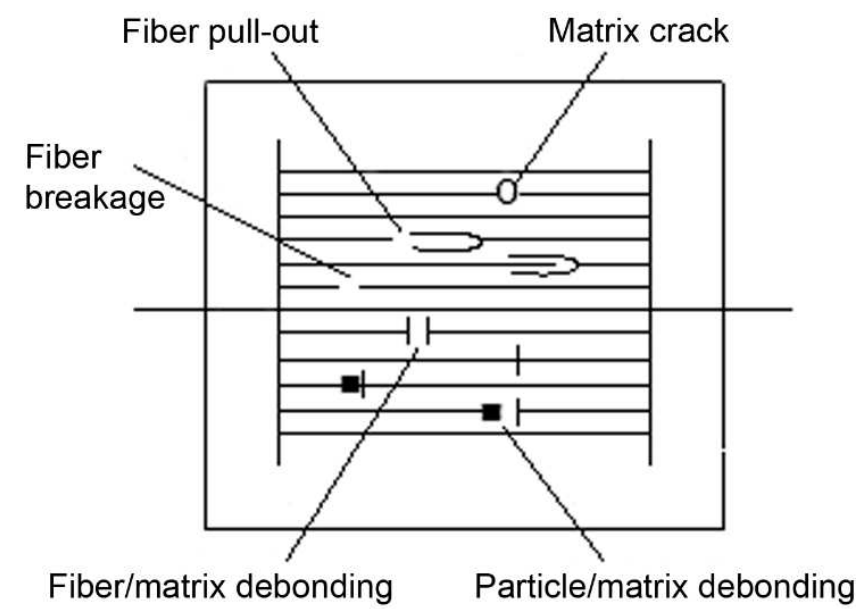

Figure 4. Bundle of fibers representing different failure modes

Consequently, with the use of the fiber-bundle-cells modeling method, every flaw population can be modeled with a special fiber bundle corresponding to the nature of damage (e.g. fiber breakage: E-bundle, fiber pull-out: ES-bundle, etc.) and the sample can be considered as the parallel connection of these fiber bundles in which the number fractions of the bundle fibers are equal to those of the failure populations (Figure 5). In this sense, the measured stressstrain curve is a realization of the stochastic tensile force process.

For simplicity, non-linear E-bundles according to Equation (1) can be used, therefore the expected tensile force-load time (the strain or elongation, $u$, is proportional to the load time, $t$ : $u=v t$; $v$ is the extension rate) curve, that is, the weighted sum of the components is as follows:

$$
E(F(t ; c, b))=\sum_{i=1}^{r} E\left(F_{i}\left(t ; c_{i}, b_{i}\right)\right)=\sum_{i=1}^{r} p_{i} \kappa_{i}\left(t ; c_{i}\right)\left(1-Q_{\varepsilon B i}\left(t ; b_{i}\right)\right)
$$


where $p_{i}$ and $\kappa_{i}$ are the number fraction and tensile characteristic of the fibers, respectively, corresponding to the volume elements containing critical flaws. In the case of failure, they realize the $i^{\text {th }}$ type damage.

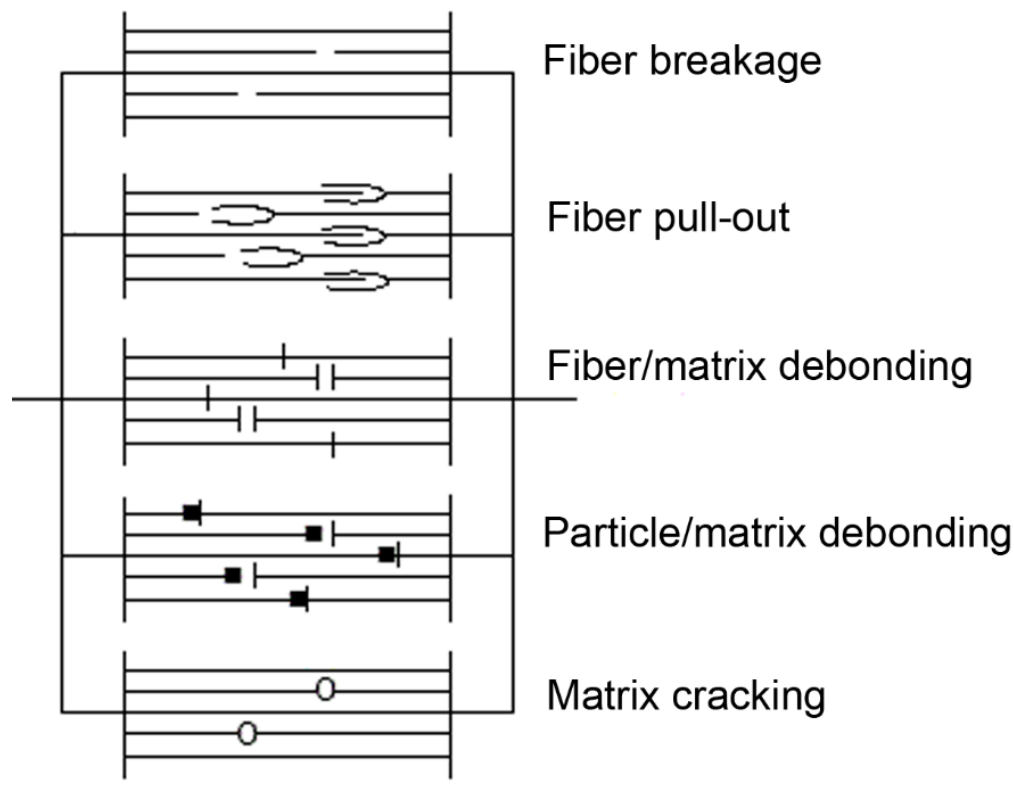

Figure 5. FBC model of fibrous structures for describing deformation and the damage process

\subsection{Decomposition of the tensile test and AE measurements using the FBC model}

\section{General case}

For the decomposition of the AE number and the tensile force processes, the parameters of the component processes corresponding to the failure modes have to be determined. They can be obtained by minimizing the squared deviation between the measured and model processes based on Equations (24), (27) and (25) and/or (26):

$$
\begin{aligned}
& \Psi_{n}^{2}=\int_{0}^{T}\left[\frac{\bar{n}(t)}{\bar{n}(T)}-\sum_{i=1}^{r} p_{i} Q_{\tau i}\left(t ; b_{i}\right)\right]^{2} d t \rightarrow \min \\
& \Psi_{F}^{2}=\int_{0}^{T}\left[\bar{F}(t)-\sum_{i=1}^{r} p_{i} \kappa_{i}\left(t ; c_{i}\right)\left(1-Q_{\tau i}\left(t ; b_{i}\right)\right)\right]^{2} d t \rightarrow \min \\
& \Psi_{w}^{2}=\int_{0}^{W}\left[\hat{Q}_{w}(x)-\sum_{i=1}^{r} p_{i} Q_{w i}\left(x ; d_{i}\right)\right]^{2} d x \rightarrow \min
\end{aligned}
$$

where $T$ is the observational time during the tensile test, $\bar{n}(T)$ is the measured number of AE events at $T, \bar{F}(t)$ is the measured mean tensile force, and $w \in\left\{\alpha, U^{+}, U, U_{E}, U_{A}\right\}$ is the selected energy property, and $W$ is the amplitude or energy limit observed. In some cases, it is worth starting with fitting the tensile characteristics to the initial part of the measured force-time curve that in the interval $\left[0, T_{0}\right]\left(0<T_{0}<T: \bar{n}\left(T_{0}\right) \ll \bar{n}(T)\right)$ is:

$$
\Psi_{F 0}^{2}=\int_{0}^{T_{0}}\left[\bar{F}(t)-\sum_{i=1}^{r} p_{i} \kappa_{i}\left(t ; c_{i}\right)\right]^{2} d t \rightarrow \min
$$


It should be noted that in general, it may be important to recognize the known sequence of the possible failure modes as a condition for fitting, which can be formulated as a series of relation for the expected value of the life times (or strength):

$$
E\left(\tau_{1}\right) \leq E\left(\tau_{2}\right) \ldots \leq E\left(\tau_{r}\right)
$$

According to experiences in the literature ${ }^{10,14}$, a similar relationship may be formulated for the expected value of the signal amplitude and energy as well.

The number of independent parameters, $I$, to be assessed from measurements, may be rather high. In the case of $r=5$ different failure modes, this means that $I$ could be larger than 30 . Thus, in general the calculations may need suitable iterative algorithms.

A possible method is firstly to determine the parameters $p_{i}$ and $b_{i}(i=1, \ldots, r)$ from Equation (28) with the help of the AE event number information, then $c_{i}$ and $d_{i}$ from Equations (29) and (30), respectively. In another case, the parameters $p_{i}$ and $c_{i}$ as preliminary information are obtained from fitting the sum of the weighted tensile characteristics $p_{i} \kappa_{i}(\mathrm{i}=1, \ldots, \mathrm{r})$ to the initial part of the measured curve with the use of Equation (31). Decomposing the measured force-strain curve and AE event number data with the help of Equations (28) and (29) provides parameters $b_{i}$ and a more correct value of $p_{i}$ and $c_{i}$. Then Equations (30) is used for determining parameters $d_{i}(i=1, \ldots, r)$. Finally, all the parameters can be estimated at the same time by minimizing the sum of the three squared deviations after normalizing Equation (29) with a possible maximum force value. In general, these calculations are based on iterative procedures at which it is important to use proper initial parameter values and approximate functions for fitting.

\section{Functions for approximation}

A linear combination of a homogeneous linear function and a finite exponential function can be applied to the mathematical form of the tensile characteristics of components. It has only three parameters, yet it can model tensile characteristics with a convex or concave initial part as well $(i=1, \ldots, r ; t>0)$ :

$$
\kappa_{i}\left(t ; c_{i}\right)=c_{1 i} t+c_{2 i}\left(1-e^{-c_{3 i} t}\right) \sim\left\{\begin{array}{c}
c_{0 i} t, t \sim 0 \\
c_{2 i}+c_{1 i} t, t \sim \infty
\end{array}\right.
$$

where $\kappa_{i}\left(t ; c_{i}\right) \geq 0$ hence $c_{1 i}, c_{3 i}>0$, but $c_{2 i}$ may be negative. The left hand side of Equation (33) presents the asymptotic expressions and $c_{0 i}$ is the initial tensile stiffness:

$$
c_{0 i}=c_{1 i}+c_{2 i} c_{3 i} \geq 0
$$

When $c_{2 i}>0$, the initial part of $\kappa_{i}$ is concave from below, while $c_{2 i}<0$ makes it convex.

When seeking the optimum according to Equation (29), it can be advantageous to reduce the number of parameters to be determined as follows:

$$
p_{i} \kappa_{i}(x)=p_{i} c_{1 i} t+p_{i} c_{2 i}\left(1-e^{-c_{3 i} t}\right)=K_{1 i} t+K_{2 i}\left(1-e^{-c_{3 i} t}\right)
$$


where $K_{1 i}$ and $K_{2 i}$ are defined by Equation (35).

In general, the damage or failure processes are governed by the minimum properties of the building elements, hence the Weibull type of two parameters $\left(b_{1 i}>0, b_{2 i} \geq 1\right)$ can be used as the distribution function of the lifetime $\left(\tau_{i j}\right):^{63}$

$$
Q_{\tau_{i}}(t)=P\left(\tau_{i j}<t\right)=1-e^{-\left(\frac{t}{b_{1 i}}\right)^{b_{2 i}}}, \quad t \geq 0
$$

The expected value and standard deviation of this distribution are given by:

$$
\begin{gathered}
E\left(\tau_{i j}\right)=b_{1 i} \Gamma\left(1+\frac{1}{b_{2 i}}\right) \\
D\left(\tau_{i j}\right)=b_{1 i} \sqrt{\Gamma\left(1+\frac{2}{b_{2 i}}\right)-\Gamma^{2}\left(1+\frac{1}{b_{2 i}}\right)}
\end{gathered}
$$

In a lot of cases, Equation (36) can be applied to describe the distribution of the signal amplitude and/or energy as well.

As a simplified case, it may be assumed that the tensile characteristic by Equation (33) is the same for all the model fibers, disregarding which failure modes they belong to. Consequently, parameter vectors $c_{i}$ are identical: $c_{i}=c(i=1, \ldots, r)$, therefore $\kappa_{i}\left(t ; c_{i}\right)=\kappa(t ; c)$. Hence, Equation (29) changes:

$$
\Psi_{F}^{2}=\int_{0}^{T}\left[\bar{F}(t)-\kappa(t ; c)\left(1-\sum_{i=1}^{r} p_{i} Q_{\tau i}\left(t ; b_{i}\right)\right)\right]^{2} d t \rightarrow \min
$$

\section{Reliability and damage maps}

In the knowledge of the components, some very informative diagrams can be plotted that characterize the reliability and the damage and failure process of the fiber-reinforced or filled sample.

The partial weighted sums of the component lifetime distributions $\left(Q_{\tau i}\right)$ show the number fraction of failures of different types detected as $\mathrm{AE}$ hits at a given time or time-proportional strain load $(k=1,2, \ldots, r)$ :

$$
0 \leq Q_{\tau k}\left(t ; B_{k}\right)=\sum_{i=1}^{k} p_{i} Q_{\tau i}\left(t ; b_{i}\right) \underset{t \rightarrow \infty}{\longrightarrow} 1
$$

where $B_{k}$ is the parameter matrix created from parameter vectors $b_{1}, \ldots, b_{k}$. Anyway, $Q_{\tau r}\left(t ; B_{r}\right)$ can be considered the distribution of the general lifetime $(\tau)$ of the sample. The plot of the curves $Q_{\tau r}\left(t ; B_{r}\right)$ determines time-dependent ranges the vertical section of which gives the number fraction of the failed model fibers at a certain load. A similar plot can be obtained if the partial weighted sums of the component reliability functions $\left(R_{i}\right)(k=1, \ldots, r)$ are calculated:

$$
1 \geq R_{k}\left(t ; B_{k}\right)=\sum_{i=1}^{k} p_{i} R_{i}\left(t ; b_{i}\right)=\sum_{i=1}^{k} p_{i}\left[1-Q_{\tau i}\left(t ; b_{i}\right)\right] \underset{t \rightarrow \infty}{\longrightarrow} 0
$$


Hence $R_{r}\left(t ; B_{r}\right)$ is the general reliability function that provides the number fraction of $i^{\text {th }}$-type fibers intact at the given load.

Besides the number of the intact fibers, their resistance force is taken into account as well, as the component tensile force-time relationships are applied as follows $(k=1, \ldots, r)$ :

$$
0 \leq F_{k}\left(t ; B_{k}\right)=\sum_{i=1}^{k} p_{i} F_{i}\left(t ; b_{i}, c_{i}\right)=\sum_{i=1}^{k} p_{i} \kappa_{i}\left(t ; c_{i}\right)\left(1-Q_{\tau i}\left(t ; b_{i}\right)\right) \underset{t \rightarrow \infty}{\longrightarrow} 0
$$

Equation (40) can be normalized with the total sum of the components when $k=r$. This ratio is the estimation of the fractions of the AE event number in the model, which characterizes the damage modes $(k=1, \ldots, r)$ :

$$
0 \leq P_{k}\left(t ; B_{k}\right)=\frac{\sum_{i=1}^{k} p_{i} Q_{\tau i}\left(t ; b_{i}\right)}{\sum_{i=1}^{r} p_{i} Q_{\tau i}\left(t ; b_{i}\right)} \underset{t \rightarrow \infty}{\longrightarrow} 0
$$

The normalization of Equation (42) by the resultant tensile force gives the ratio of the tensile force components as a fraction of the resultant force at the current time (strain load). $(k=1, \ldots, r)$ :

$$
0 \leq P_{F k}\left(t ; B_{k}\right)=\frac{\sum_{i=1}^{k} p_{i} F_{i}\left(t ; b_{i}, c_{i}\right)}{\sum_{i=1}^{r} p_{i} F_{i}\left(t ; b_{i}, c_{i}\right)}=\frac{\sum_{i=1}^{k} p_{i} \kappa_{i}\left(t ; c_{i}\right)\left(1-Q_{\tau i}\left(t ; b_{i}\right)\right)}{\sum_{i=1}^{r} p_{i} \kappa_{i}\left(t ; c_{i}\right)\left(1-Q_{\tau i}\left(t ; b_{i}\right)\right)} \underset{t \rightarrow \infty}{\longrightarrow} 1
$$

$P_{F k}$ characterizes both the current resistance of the sample and the failure process as well because it expresses the tensile force fraction of the fibers intact at the current time.

The curve families create a kind of failure map where the ranges between the curves characterize the participation of the components and the failure processes represented by them. This is vividly descriptive when the curves $R_{k}, P_{F k}$ or $P_{k}(k=1, \ldots, r)$, providing percentages are used.

\section{Experimental}

In order to demonstrate the applicability of the statistical fiber-bundle-cells model, we tested two types of fiber-reinforced polypropylene composites with different failure processes and analyzed the tensile data and the $\mathrm{AE}$ measurement, and characterized the reliability and damage modes. The failure process of either composite was gradual and most of the introduced relationships were applied to evaluation, while in the case of the other composite, the ultimate failure was catastrophic and the effect of the coupling agent could be discussed based on FBC modeling and the reliability and damage map results. In all cases, single measurements were decomposed and analyzed based on the expected value of the modeled processes.

\subsection{Glass fiber reinforced PP composite}

CT Compact Tension specimens were manufactured from VERTON polypropylene sheets reinforced by $\sim 20 \mathrm{~m} \%$ ( $\sim 8 \mathrm{~V} \%$ ) short glass fibers (PP/SGF) (produced by ICI Great Britain), according to ASTM Standard D5045 (Standard Test Methods for Plane-Strain Fracture 
Toughness and Strain Energy Release Rate of Plastic Materials). ${ }^{14}$ The length $(l)$ and aspect ratio $(l / d)$ of fibers were $240-600 \mu \mathrm{m}$ and 20-50 respectively. Tensile tests were carried out on a Zwick 1445 universal tester with a rate of elongation of $v=1 \mathrm{~mm} / \mathrm{min}$ at an ambient temperature of $22^{\circ} \mathrm{C}$. The tensile force $(F)$ and elongation $(u=v t)$ were recorded. AE measurements were performed with Defectophone NEZ-220 (produced by KFKI, Hungary) with a DAE-002 microphone (bandwidth: 20-1000 kHz). ${ }^{14}$ The measured AE signals were evaluated after filtering out the background noise and noise caused by the tester and gripping. Figure 6 shows the results of the tensile test and AE measurements, which are the tensile force vs. time (vertical blue dotted line indicates the force peak) and the AE event number vs. time records.

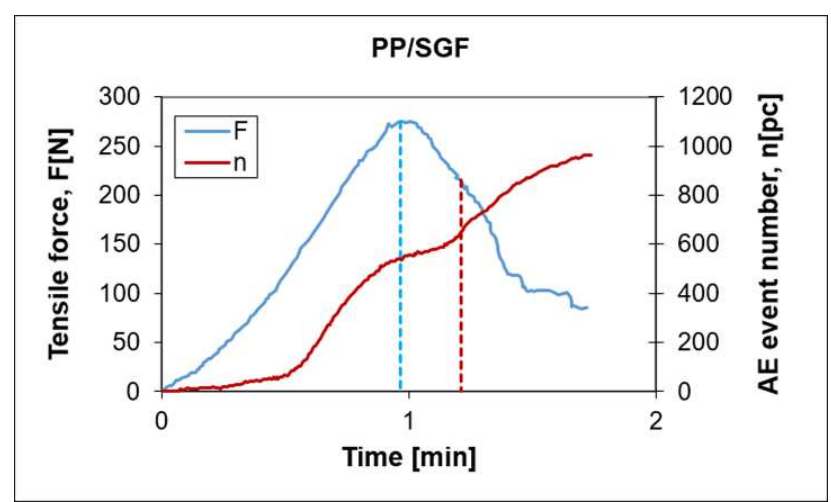

Figure 6. Measured tensile force-time curve and cumulative event number process of PP composite reinforced with short glass fibers

Besides the AE event number, the amplitude and energy values were evaluated in the interval limited by a displacement $\left(u_{A E}=v t_{A E}\right)$ or test time $\left(t_{A E}=0.7 \cdot T \approx 1.2 \mathrm{~min}\right)$ value, providing a load where all the failure modes were detected (vertical red dotted line in Figure 6: $t_{A E}$ ) beyond the tensile force peak for the sake of comparing the ranges including the usual loads. Table 1 contains the measured event number $(n)$ (pcs=pieces) and tensile test $(u, F)$ data belonging to the observation time $(T)$ and the force peak time $\left(t^{*}\right)$.

Table 1. Measured properties of the composites tested

\begin{tabular}{|c|c|c|c|c|c|c|c|}
\hline Composite & $\begin{array}{c}\mathbf{T} \\
{[\mathbf{m i n}]}\end{array}$ & $\begin{array}{c}\mathbf{n}(\mathbf{T}) \\
{[\mathbf{p c s}]}\end{array}$ & $\begin{array}{c}\mathbf{t}^{*} \\
{[\mathbf{m i n}]}\end{array}$ & $\begin{array}{c}\mathbf{u}^{*} \\
{[\mathbf{m m}]}\end{array}$ & $\begin{array}{c}\mathbf{F}^{*} \\
{[\mathbf{N}]}\end{array}$ & $\begin{array}{c}\mathbf{u}(\mathbf{T}) \\
{[\mathbf{m m}]}\end{array}$ & $\begin{array}{c}\mathbf{F}(\mathbf{T}) \\
{[\mathbf{N}]}\end{array}$ \\
\hline SGF/PP & 1.72 & 964 & 0.965 & 0.965 & 275 & 1.72 & 85.3 \\
\hline
\end{tabular}

Based on serial microphotographs of crack growth during loading and AE signal analysis at different loads, as well as SEM micrographs taken of the fracture surface, four possible damage modes were identified and chronologically ordered: local matrix deformation and damage (craze and microcrack), fiber-matrix debonding, fiber pullout, and fiber breakage. ${ }^{10,14}$ Because of the short fibers, the damage modes of small energy release (matrix damage, debonding) dominated the failure process and the AE signals were of larger amplitude and energy indicating damage modes connected to fibers (pull-out, breakage) (Figure 7).

Consequently, the four damage modes listed above were considered significant to model for both composites tested $(r=4)$, which were denoted by D1,..,D4. 
Nonlinear tensile characteristics by Equations (33)-(35) were used for the force components since the initial part of the measured force vs. time curves (Figure 6) is convex from below.

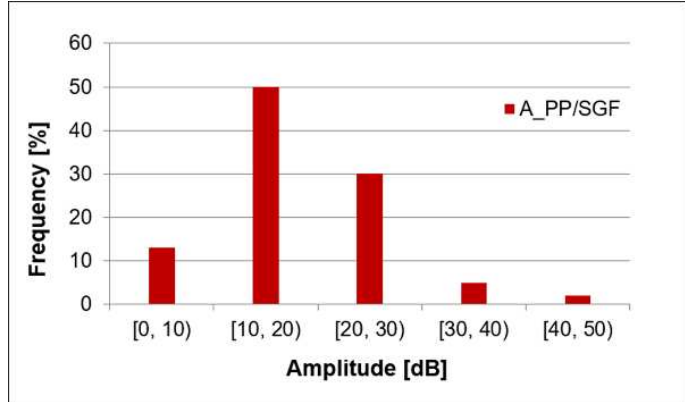

(a)

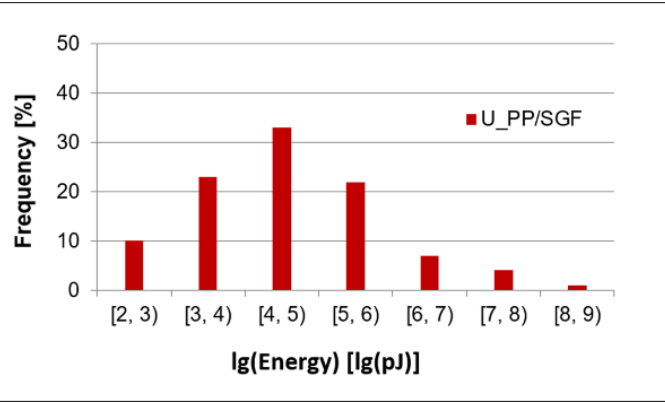

(b)

Figure 7. Frequency histograms of signal amplitudes (a) and the energy (b) of PP/SGF composites

The parameters of the components (Table 2) were determined from fitting their weighted sum to the measurements. Fitting was carried out in the following steps:

(1) Minimizing Equation (31) provided the preliminary values of parameters $p_{\mathrm{i}}, c_{3 i}$, and $K_{\mathrm{ji}}=p_{\mathrm{ji}} c_{\mathrm{ji}}(j=1,2 ; i=1, \ldots, r)$ of the component tensile force-time relations (see Equation (41)). These parameters were used as initial values in the sequence.

(2) Minimizing Equation (28) and using Weibull distribution by Equation (36) for the component lifetimes $\tau_{i}$ provided parameters $p_{\mathrm{i}}$ and $b_{i}(i=1, \ldots, r)$ of the component AE event processes.

(3) Minimizing Equation (29) provided parameters $c_{\mathrm{i}}(i=1, \ldots, r)$ of the component tensile characteristics.

(4) Minimizing Equation (30) and checking the results with a khi-squared test provided parameters $d_{\mathrm{i}}(i=1, \ldots, r)$ of the signal amplitude (and/or energy) distribution components.

The results of the decomposition of the $\mathrm{AE}$ event number $(n)$ and the tensile force $(F) \mathrm{vs}$. time $(t)$ processes can be seen in Figure 8.

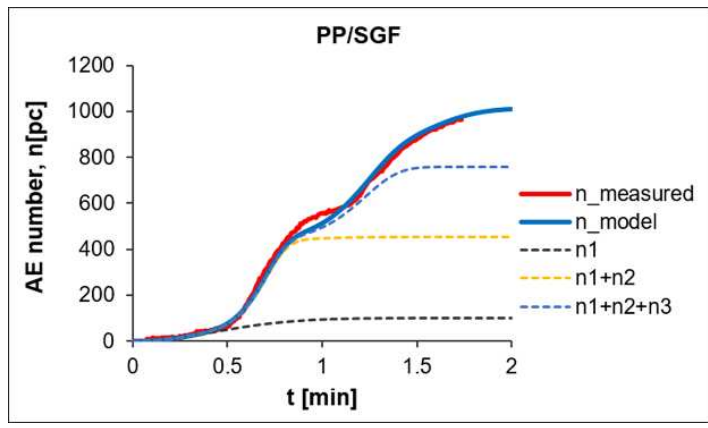

(a)

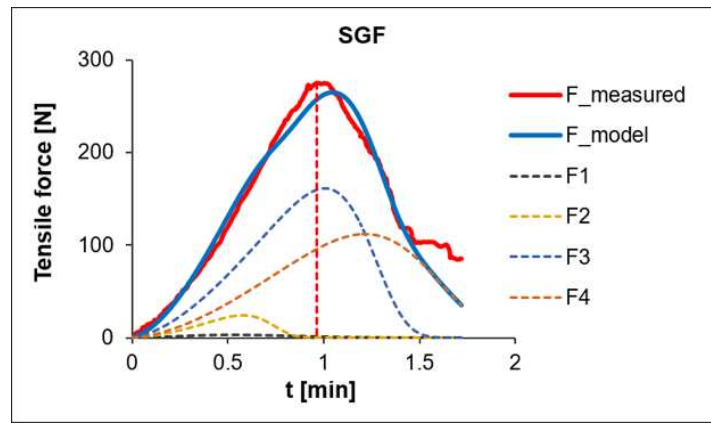

(b)

Figure 8. The measured and modeled cumulative event number process (a) and tensile forcetime curve (b) of the PP/SGF composite

Table 2 shows the component parameters of the event number and the tensile characteristic obtained from fitting where $n_{\infty}$ and $p_{\infty}$ are the asymptotic values of the event number and the 
failure type fraction respectively. The relative mean squared error (MSE) related to the maximum of the measured value was less than $4.3 \%$ in every case regarding both the event number $(\operatorname{MSE}(n))$ and the tensile force $(\operatorname{MSE}(F))$.

Table 2. Parameters of the model components (MCi) and the resultant model (M) obtained from fitting

\begin{tabular}{|c|c|c|c|c|c|}
\hline \multirow{2}{*}{ Parameters } & \multicolumn{5}{|c|}{ SGF/PP } \\
\hline & MC1 & MC2 & MC3 & MC4 & $\mathbf{M}$ \\
\hline$n_{\infty i}[p c s]$ & 101 & 353 & 303 & 253 & 1010 \\
\hline$p_{\infty i}[-]$ & 0.10 & 0.35 & 0.30 & 0.25 & 1.00 \\
\hline $\mathbf{b}_{1 \mathrm{i}}[\mathrm{min}]$ & 0.60 & 0.72 & 1.25 & 1.55 & \\
\hline $\mathbf{b}_{2 \mathrm{i}}[-]$ & 2.00 & 7.00 & 8.00 & 6.00 & \\
\hline MSE (n) & \multicolumn{5}{|c|}{$1.93 \%$} \\
\hline $\mathrm{c}_{1 \mathrm{i}}[\mathrm{N} / \mathrm{min}]$ & 300 & 300 & 1000 & 700 & 2300 \\
\hline $\mathrm{c}_{2 \mathrm{i}}[\mathrm{N}]$ & -120 & -120 & -410 & -310 & -250 \\
\hline $\mathrm{c}_{3 \mathrm{i}}[1 / \mathrm{min}]$ & 2.30 & 2.30 & 2.20 & 2.10 & 2.23 \\
\hline $\mathrm{c}_{0 \mathrm{i}}[\mathrm{N} / \mathrm{min}]$ & 24 & 24 & 98 & 49 & 195 \\
\hline $\mathrm{K}_{1 \mathrm{i}}[\mathbf{N} / \mathrm{min}]$ & 30 & 105 & 300 & 175 & 610 \\
\hline $\mathrm{K}_{\mathbf{0 i}}[\mathrm{N} / \mathrm{min}]$ & 2.40 & 8.40 & 29.40 & 12.25 & 52.45 \\
\hline $\operatorname{MSW}(\mathbf{F})$ & \multicolumn{5}{|c|}{$4.22 \%$} \\
\hline
\end{tabular}

Table 3 contains some special values of the event number and the fraction of the failure modes calculated for the duration of measuring $(T)$ and analyzing the AE signal amplitude and energy $\left(t_{A E}=0.7 \cdot T\right)$. It also shows the descriptive statistics of the lifetime $\left(\tau_{\mathrm{i}}\right)$ characterizing the different failure modes (E: expected value, $\mathrm{D}$ : standard deviation, V: relative standard deviation). and that the expected lifetime values $\left(\mathrm{E}\left(\tau_{\mathrm{i}}\right)\right)$ of the components satisfy the condition according to Equation (32). Finally, $t_{q i} *$ is the lifetime value belonging to the peak of the density function of $\tau_{i}$.

Table 3. Calculated properties of the model components and damage modes (MCi) and the resultant model (M)

\begin{tabular}{|c|c|c|c|c|c|}
\hline \multirow{2}{*}{ Parameters } & \multicolumn{5}{|c|}{ SGF/PP } \\
\cline { 2 - 6 } & MC1 & MC2 & MC3 & MC4 & M \\
\hline $\mathbf{n}_{\infty \mathbf{i}}[\mathbf{p c s}]$ & 101 & 353 & 303 & 253 & 1010 \\
\hline $\mathbf{p}_{\infty \mathbf{o i}}[-]$ & 0.10 & 0.35 & 0.30 & 0.25 & 1.00 \\
\hline $\mathbf{n}_{\mathbf{i}}(\mathbf{T})[\mathbf{p c}]$ & 101 & 354 & 303 & 213 & 971 \\
\hline $\mathbf{p}_{\mathbf{i}}(\mathbf{T})[-]$ & 0.10 & 0.37 & 0.31 & 0.22 & 1.00 \\
\hline $\mathbf{n}_{\mathbf{i}}\left(\mathbf{t}_{\mathbf{A E}}\right)[\mathbf{p c}]$ & 99 & 354 & 163 & 51 & 667 \\
\hline $\mathbf{p}_{\mathbf{i}}\left(\mathbf{t}_{\mathbf{A E}}\right)[-]$ & 0.15 & 0.53 & 0.24 & 0.08 & 1.00 \\
\hline $\mathbf{t}_{\mathbf{q i}}{ }^{*}[\mathbf{m i n}]$ & 0.42 & 0.70 & 1.23 & 1.50 & \\
\hline $\mathbf{E}\left(\tau_{\mathbf{i}}\right)[\mathbf{m i n}]$ & 0.53 & 0.67 & 1.18 & 1.44 & 1.00 \\
\hline $\mathbf{E}\left(\tau_{\mathbf{i}}{ }^{2}\right)\left[\mathbf{m i n}{ }^{2}\right]$ & 0.36 & 0.47 & 1.42 & 2.15 & 1.16 \\
\hline $\mathbf{D}\left(\tau_{\mathbf{i}}\right)[\mathbf{m i n}]$ & 0.28 & 0.11 & 0.17 & 0.28 & 0.40 \\
\hline $\mathbf{V}\left(\tau_{\mathbf{i}}\right)[\%]$ & 52.3 & 16.8 & 14.8 & 19.4 & 39.6 \\
\hline
\end{tabular}


The decomposition results make it possible to calculate the reliability and failure mode characteristics according to Equations (41)-(44). Figure 9 shows the fraction of the AE events according to Equation (43) belonging to the four damage modes (D1,..,D4) at different load times. The vertical dotted blue and red lines indicate the time of the peak force $\left(t^{*}\right)$ and the upper limit $\left(t_{A E}\right)$ of the duration of detecting the signal amplitude and energy respectively.

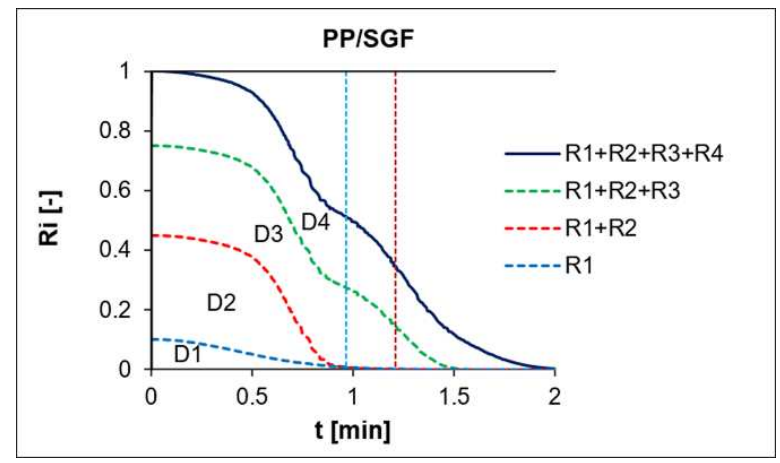

Figure 9. The component and resultant reliability functions of the PP/SGF composite sample belonging to different damage modes $(\mathrm{D} i)$ as a function of load time

Figure 10.a shows the calculated fractions of the AE events given by Equation (43) belonging to the four damage modes (D1,..,D4) at different load times while Figure 10.b shows the fractions of the component and resultant force resistances according to Equation (44) provided by the intact model fibers belonging to the different damage modes.

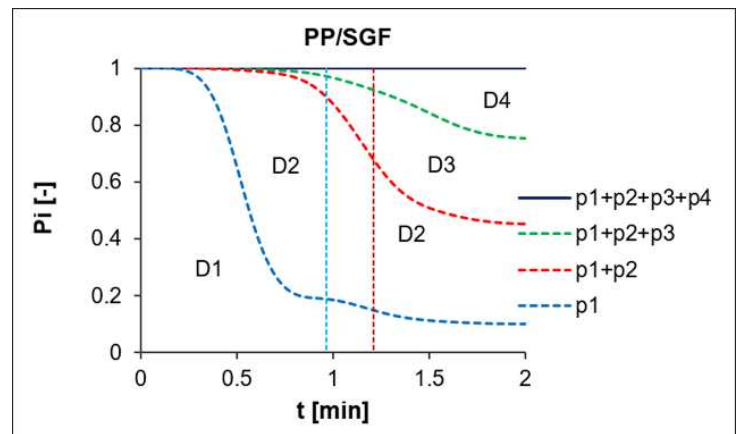

(a)

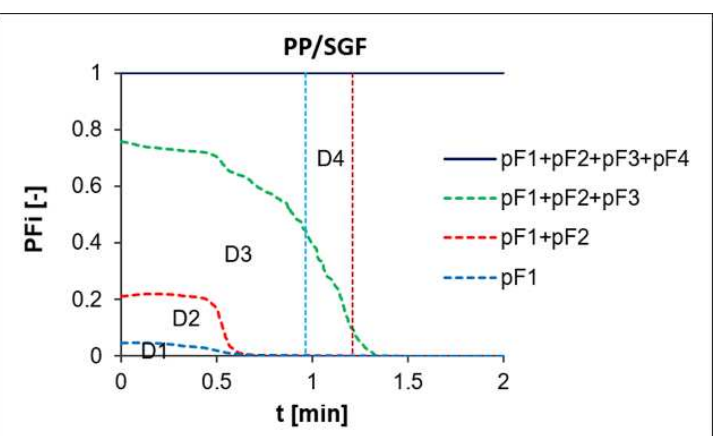

(b)

Figure 10. Fraction of AE event number (a) and force resistance (b) belonging to different damage modes $(\mathrm{Di})$ as a function of the load time

It is observable in Figure 10 that although the damage of fibers of D1 and D2 types (matrix failures and debonding) lasts during the test (Figure 10.a), their resistance becomes essentially negligible beyond the peak time related to that of fibers of D3 and D4 types representing the slippage and breakage of fibers (Figure 10.b).

Probability distributions of signal amplitude and energy are characterized by the frequency histograms in Figures 11 measured up to the time $t_{A E}=0.7 \cdot T$ (beyond the force peak) and the probability density function of the fitted components in Figures 12.

The parameters and calculated properties of the component and fitted resultant distribution are contained in Table 4. Fitting could be carried out with small relative mean squared error $(\mathrm{MSE}<2.3 \%)$ and according to the khi-squared test, the agreement between the measured and 
modeled histograms is highly acceptable (amplitude: $p$-value $=0.411$, energy: $p$-value $=0.810$ ) (Figures 11, 12).

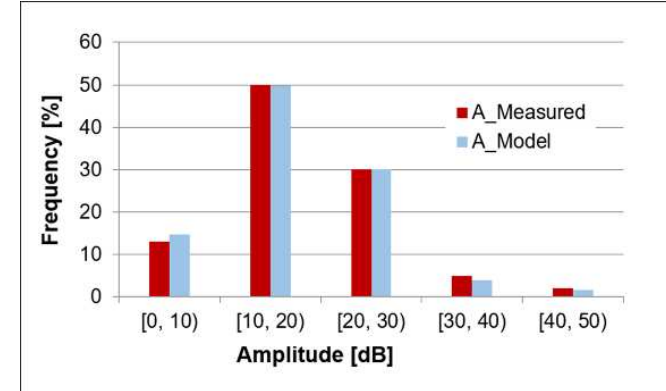

(a)

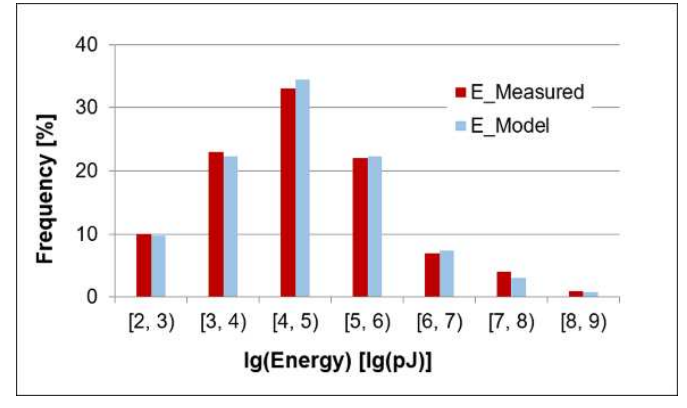

(b)

Figure 11. Signal amplitude (a) and energy (b) histograms measured and modeled for the PP/SGF composite

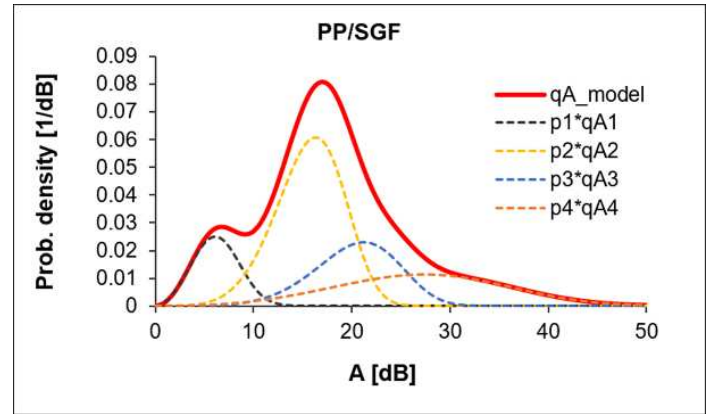

(a)

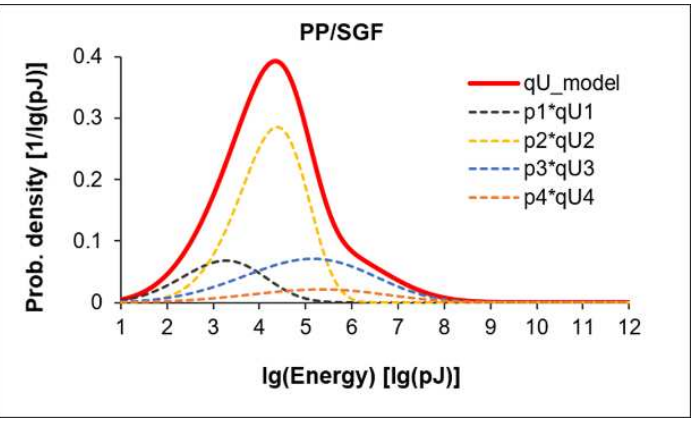

(b)

Figure 12. Fitted probability density functions of the signal amplitude (a) and energy (b) and their components for the PP/SGF composite

Table 4. Parameters of the component distributions of the signal amplitude and energy obtained from fitting

\begin{tabular}{|c|c|c|c|c|c|}
\hline \multirow{2}{*}{ Parameters } & \multicolumn{5}{|c|}{ SGF/PP } \\
\hline & MC1 & MC2 & MC3 & MC4 & $\mathbf{M}$ \\
\hline $\mathbf{n}_{\mathbf{i}}\left(\mathbf{t}_{\mathrm{AE}}\right)[\mathrm{pc}]$ & 99 & 354 & 163 & 51 & 667 \\
\hline $\mathbf{p}_{\mathbf{i}}\left(\mathbf{t}_{\mathrm{AE}}\right)[-]$ & 0.149 & 0.530 & 0.244 & 0.077 & 1.00 \\
\hline$d_{1 i}[d B]$ & 7.8 & 18.8 & 24.0 & 35.0 & \\
\hline$d_{2 i}[-]$ & 3.0 & 6.0 & 5.0 & 3.6 & \\
\hline$A_{q i} *[d B]$ & 6.81 & 18.24 & 22.95 & 31.97 & \\
\hline$E\left(A_{i}\right)[d B]$ & 6.97 & 17.44 & 22.04 & 31.54 & 18.09 \\
\hline$D\left(A_{i}\right)[d B]$ & 2.53 & 3.38 & 5.05 & 9.73 & \\
\hline $\mathbf{V}\left(\mathbf{A}_{\mathbf{i}}\right)[\%]$ & 0.36 & 0.19 & 0.23 & 0.31 & \\
\hline $\operatorname{MSE}(q A)$ & \multicolumn{5}{|c|}{$1.08 \%$} \\
\hline$d_{1 i}[\lg (p J)]$ & 3.5 & 4.8 & 5.8 & 6.0 & \\
\hline $\mathbf{d}_{2 \mathrm{i}}[-]$ & 4.8 & 6.7 & 4.2 & 4.2 & \\
\hline $\mathrm{U}_{\mathrm{qi}}{ }^{*}[\lg (\mathrm{pJ})]$ & 3.33 & 4.69 & 5.44 & 5.62 & \\
\hline$E\left(U_{i}\right)[\lg (p J)]$ & 3.21 & 4.48 & 5.27 & 5.45 & 4.56 \\
\hline $\mathbf{D}\left(\mathbf{U}_{\mathrm{i}}\right)[\lg (\mathbf{p J})]$ & 0.76 & 0.78 & 1.42 & 1.46 & \\
\hline $\mathbf{V}\left(\mathbf{U}_{\mathbf{i}}\right)[\%]$ & 0.24 & 0.17 & 0.27 & 0.27 & \\
\hline $\operatorname{MSE}(q \mathbf{U})$ & \multicolumn{5}{|c|}{$2.25 \%$} \\
\hline
\end{tabular}


It should be noted that the expected values of both the amplitude $\left(\mathrm{E}\left(A_{i}\right)\right)$ and the energy $\left(\mathrm{E}\left(U_{i}\right)\right)$ (Table 4) satisfy the condition similar to Equation (32), that is, $\mathrm{E}\left(X_{i}\right)<\mathrm{E}\left(X_{i+1}\right)(X=A$ or $U ; i=1,2,3)$ or in other words, a larger expected failure energy belongs to larger expected lifetime.

\subsection{Wood fiber reinforced PP composites}

Dumbbell composite specimens (cross section area: $4 \mathrm{~mm} \times 10 \mathrm{~mm}=40 \mathrm{~mm}^{2}$ ) were prepared with an Arburg $420 \mathrm{C}$ Advance 1000-250 injection molding machine from PP/wood fiber (PP/WF) blends produced with a Brabender Plasticorder twin-screw extruder. ${ }^{60}$ The polypropylene was Tipplen H-116 homopolymer (TVK, Hungary, density: $\rho=0.9 \mathrm{~g} / \mathrm{cm}^{3}$ ) and the wood fiber was obtained from sawmill waste of beech wood (Furnér Müvek Kft., Budapest, Hungary) with the use of a sieve shaking machine. The wood particles (dried at $105^{\circ} \mathrm{C}$ for $24 \mathrm{~h}$ ) were a longish bundle of elementary fibers the size of which was less than 1 $\mathrm{mm}$ (dominant length class: $0.4-0.6 \mathrm{~mm}$, density $\rho \sim 0.8 \mathrm{~g} / \mathrm{cm}^{3}$ ). After manufacturing, the dominant length class of wood fibers was reduced to 0.15-0.20 mm. PPgMA (Licomont AR 504 FG, Clariant, Germany; $\rho=0.91 \mathrm{~g} / \mathrm{cm}^{3}$ ) was used as coupling agent. The composition of the specimens used for testing is shown in Table 5.

Table 5. Constituents of composites

\begin{tabular}{|c|c|c|c|c|c|}
\hline \multirow{2}{*}{ Component } & Density & \multicolumn{2}{|c|}{ Composite 1 } & \multicolumn{2}{c|}{ Composite 2 } \\
\cline { 2 - 6 } & {$\left[\mathrm{g} / \mathrm{cm}^{3}\right]$} & $\mathrm{m} \%$ & $\mathrm{~V} \%$ & $\mathrm{~m} \%$ & $\mathrm{~V} \%$ \\
\hline PP & 0.90 & 60.0 & 57.1 & 58.0 & 55.2 \\
\hline Wood fiber & 0.80 & 40.0 & 42.9 & 40.0 & 42.9 \\
\hline PPgMA & 0.91 & & & 2.0 & 1.9 \\
\hline
\end{tabular}

Tensile tests according to the ISO 527 Standard were carried out with a Zwick Z005 universal tester at a rate of elongation $\nu=20 \mathrm{~mm} / \mathrm{min}$ and gauge length $l_{0}=115$ and ambient temperature $T=22^{\circ} \mathrm{C} .{ }^{60}$ Elongation was measured in the middle of the samples with a Zwick BW 40220 video-extensometer. AE measurements were performed with a Sensophone AED 40/12 (Gereb \& Co Ltd., Hungary) with a Micro 30S piezoelectric sensor (Physical Acoustic Corporation, USA). The bandwidth and threshold were $100-600 \mathrm{kHz}$ and $26 \mathrm{~dB}$ respectively. By contrast to the PP/glass fiber (PP/SGF) composite (Chapter 3.1), the PP/wood fiber (PP/WF) composite samples broke suddenly during the measurements.

Figure 13 shows some typical measurement results for the composites in Table 5. 


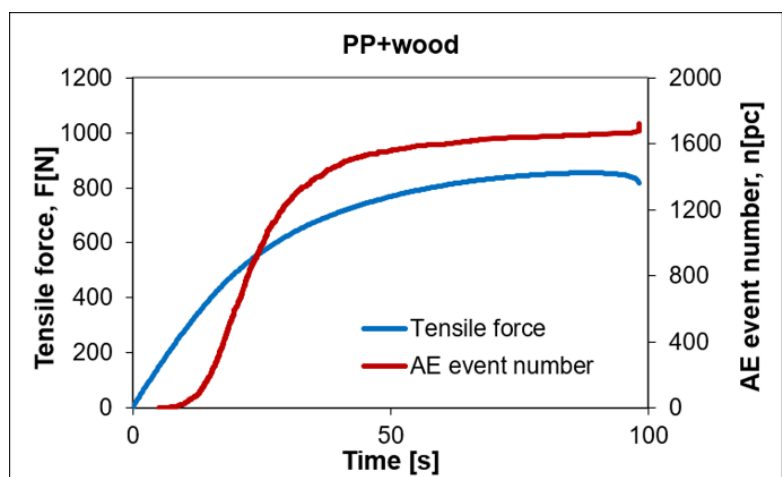

(a)

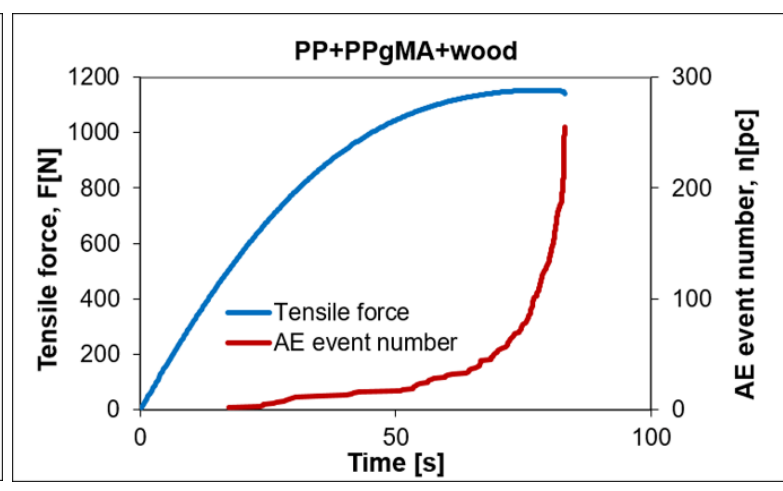

(b)

Figure 13. Tensile force-load time curve and cumulative event number process of PP/wood composite without (a) and with a coupling agent (b)

Table 6 shows some characteristic values of the tensile force $(F)$, elongation $(u)$ and AE event number $(n)$ (pcs=pieces) measured at the end of the observation time $(T)$ and at the time $t^{*}$ belonging to the force peak.

Table 6. Measured properties of the composites tested

\begin{tabular}{|c|c|c|c|c|c|c|c|}
\hline \multirow{2}{*}{ Composite } & $\mathbf{T}$ & $\mathbf{n}(\mathbf{T})$ & $\mathbf{t}^{*}$ & $\mathbf{u}^{*}$ & $\mathbf{F}^{*}$ & $\mathbf{u}(\mathbf{T})$ & $\mathbf{F}(\mathbf{T})$ \\
\cline { 2 - 8 } & {$[\mathrm{s}]$} & {$[\mathrm{pcs}]$} & {$[\mathrm{s}]$} & {$[\mathrm{mm}]$} & {$[\mathrm{N}]$} & {$[\mathrm{mm}]$} & {$[\mathrm{N}]$} \\
\hline PP+woof & 98.2 & 1718 & 89.4 & 2.98 & 854 & 3.27 & 818 \\
\hline PP+PPgMA+wood & 83.1 & 255 & 79.5 & 2.65 & 1154 & 2.77 & 1140 \\
\hline
\end{tabular}

In this case, the number of the components representing different damage modes was five $(r=5)$ because the expected value process of the FBC model used represents continuous damage and the measured tensile force vs. time curves with a broken end indicating a sudden fracture need a closing part realized by the fifth component (Figures 14 and 15).

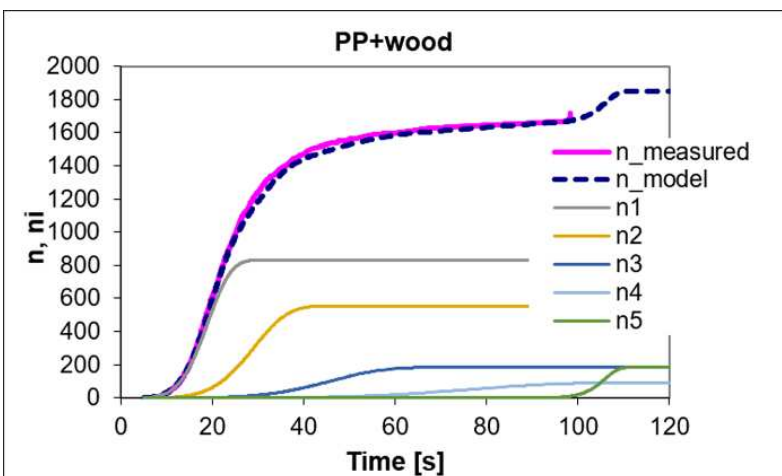

(a)

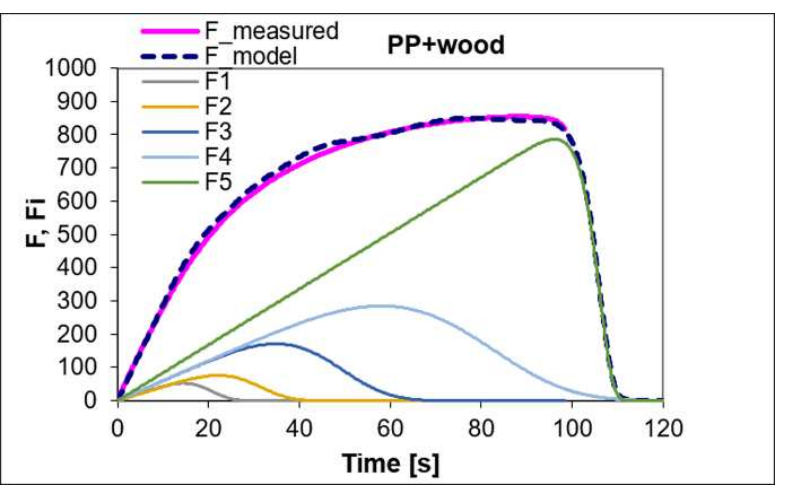

(b)

Figure 14. Measured and modeled cumulative event number process (a) and tensile forcetime curve (b) of PP/wood composite without a coupling agent 


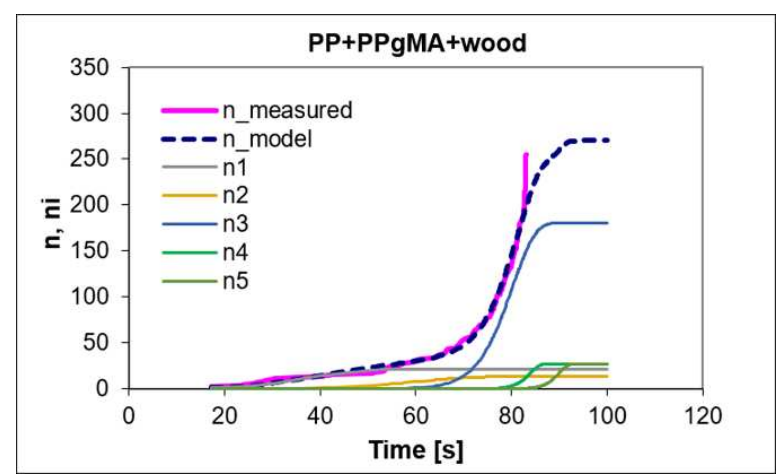

(a)

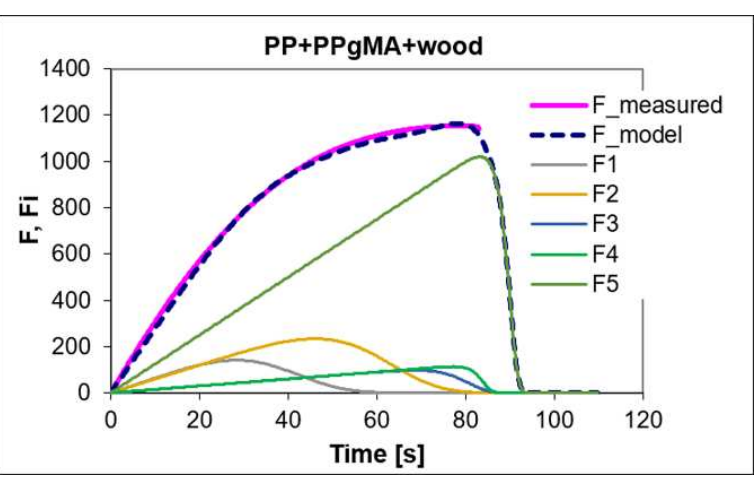

(b)

Figure 15. Measured and modeled cumulative event number process (a) and tensile forcetime curve (b) of PP/WF composite with a coupling agent (PPgMA)

Otherwise, linear tensile characteristics were used for the force components $\left(c_{1 i}>0, c_{2 i}=c_{3 i}=0\right.$, $i=1, \ldots, 5$ in Equation (33)) since the initial part of the force-time curves could be approximated with a homogeneous linear relationship.

The relative mean squared error of the FBC model fitting was less than $1.7 \%$ in three cases (Table 7) while it was $\sim 4 \%$ in the fourth case, where the AE event number increased suddenly at the end of measurement, closed by a sudden breakage, which was not modeled since it was just a single phenomenon to be averaged for the expected value model used (Figure 15.a).

Table 7. Parameters of the model components (MCi) and the resultant model (M) obtained from fitting

\begin{tabular}{|c|c|c|c|c|c|c|c|c|c|c|c|c|}
\hline \multirow{2}{*}{ Parameters } & \multicolumn{6}{|c|}{ PP+wood } & \multicolumn{6}{|c|}{ PP+PPgMA+wood } \\
\hline & MC1 & MC2 & MC3 & MC4 & MC5 & M & MC1 & MC2 & MC3 & MC4 & MC5 & M \\
\hline $\mathbf{n}_{\infty i}[\mathrm{pcs}]$ & 832 & 555 & 185 & 93 & 185 & 1850 & 22 & 13 & 181 & 27 & 27 & 270 \\
\hline$p_{\infty i}[-]$ & 0.45 & 0.30 & 0.10 & 0.05 & 0.10 & 1.00 & 0.08 & 0.05 & 0.67 & 0.10 & 0.10 & 1.00 \\
\hline $\mathbf{b}_{1 \mathrm{i}}[\mathrm{s}]$ & 20.0 & 30.5 & 48.0 & 80.0 & 106.0 & & 40.0 & 62.0 & 80.5 & 84.0 & 90.0 & \\
\hline $\mathbf{b}_{2 \mathrm{i}}[-]$ & 4.8 & 5.0 & 5.0 & 5.0 & 37.0 & & 4.0 & 6.0 & 18.0 & 45.0 & 50.0 & \\
\hline MSE(n) & \multicolumn{6}{|c|}{$1.64 \%$} & \multicolumn{6}{|c|}{$4.02 \%$} \\
\hline$c_{1 i}[N / s]$ & 10 & 14 & 60 & 120 & 84 & 288 & 80 & 120 & 2 & 15 & 125 & 342 \\
\hline $\mathbf{c}_{2 \mathrm{i}}[\mathbf{N}]$ & 0 & 0 & 0 & 0 & 0 & 0 & 0 & 0 & 0 & 0 & 0 & 0 \\
\hline$c_{3 i}[1 / s]$ & 0 & 0 & 0 & 0 & 0 & & 0 & 0 & 0 & 0 & 0 & \\
\hline $\mathrm{c}_{0 \mathrm{i}}[\mathrm{N} / \mathrm{s}]$ & 10 & 14 & 60 & 120 & 84 & 288 & 80 & 120 & 2 & 15 & 125 & 342 \\
\hline$K_{1 i}[N / s]$ & 4.5 & 4.2 & 6.0 & 6.0 & 8.4 & 29.1 & 6.4 & 6.0 & 1.5 & 1.5 & 12.5 & 27.9 \\
\hline $\mathbf{K}_{\mathbf{0 i}}[\mathbf{N} / \mathbf{s}]$ & 4.5 & 4.2 & 6.0 & 6.0 & 8.4 & 29.1 & 6.4 & 6.0 & 1.5 & 1.5 & 12.5 & 27.9 \\
\hline MSE(F) & \multicolumn{6}{|c|}{$0.67 \%$} & \multicolumn{6}{|c|}{$1.45 \%$} \\
\hline
\end{tabular}

Table 8. Calculated properties of the model components and damage modes (MCi) and the resultant model $(\mathrm{M})(\mathrm{pcs}=$ piece)

\begin{tabular}{|c|c|c|c|c|c|c|c|c|c|c|c|c|}
\hline \multirow{2}{*}{ Parameters } & \multicolumn{9}{|c|}{ PP+wood } & \multicolumn{6}{c|}{ PP+PPgMA+wood } \\
\cline { 2 - 15 } & MC1 & MC2 & MC3 & MC4 & MC5 & M & MC1 & MC2 & MC3 & MC4 & MC5 & M \\
\hline $\mathbf{n}_{\infty \mathbf{i}}[\mathbf{p c s}]$ & 832 & 555 & 185 & 93 & 185 & 1850 & 22 & 13 & 181 & 27 & 27 & 270 \\
\hline $\mathbf{p}_{\infty \mathbf{i}}[-]$ & 0.45 & 0.30 & 0.10 & 0.05 & 0.10 & 1.00 & 0.08 & 0.05 & 0.67 & 0.10 & 0.10 & 1.00 \\
\hline $\mathbf{n}_{\mathbf{i}}(\mathbf{T})[\mathbf{p c}]$ & 832 & 555 & 185 & 87 & 11 & 1670 & 22 & 13 & 13 & 150 & 1 & 199 \\
\hline $\mathbf{p}_{\mathbf{i}}(\mathbf{T})[-]$ & 0.50 & 0.33 & 0.11 & 0.05 & 0.01 & 1.00 & 0.11 & 0.07 & 0.06 & 0.75 & 0.00 & 1.00 \\
\hline \multirow{2}{*}{$\mathbf{t}_{\mathbf{q}} *[\mathbf{s}]$} & 19.05 & 29.17 & 45.90 & 76.5 & 105.9 & & 37.2 & 60.1 & 80.2 & 83.9 & 89.9 & \\
& & & & 1 & 2 & & 2 & 4 & 4 & 6 & 6 & \\
\hline \multirow{2}{*}{$\mathbf{E}\left(\tau_{\mathbf{i}}\right)[\mathbf{s}]$} & 18.32 & 28.00 & 44.07 & 73.4 & 104.4 & 35.1 & 36.2 & 57.5 & 78.1 & 82.9 & 89.0 & 75.3 \\
& & & & 5 & 2 & 7 & 6 & 2 & 5 & 6 & 0 & 3 \\
\hline
\end{tabular}




\begin{tabular}{|c|c|c|c|c|c|c|c|c|c|c|c|c|}
\hline $\mathbf{E}\left(\tau_{\mathrm{i}}^{2}\right)\left[\mathbf{s}^{2}\right]$ & 355 & 825 & 2044 & 5678 & 10916 & 1987 & 1418 & 3433 & 6137 & 6888 & 7925 & 5878 \\
\hline $\mathbf{D}\left(\tau_{\mathbf{i}}\right)[\mathbf{s}]$ & 4.36 & 6.41 & 10.09 & $\begin{array}{c}16.8 \\
2\end{array}$ & 3.55 & $\begin{array}{c}27.3 \\
9\end{array}$ & $\begin{array}{c}10.1 \\
7\end{array}$ & $\begin{array}{c}11.1 \\
5\end{array}$ & 5.36 & 2.33 & 2.25 & $\begin{array}{c}14.2 \\
4\end{array}$ \\
\hline $\mathbf{V}\left(\tau_{\mathrm{i}}\right)[\%]$ & 23.8 & 22.9 & 22.9 & 22.9 & 3.4 & 77.9 & 28.1 & 19.4 & 6.9 & 2.8 & 2.5 & 18.9 \\
\hline
\end{tabular}

The expected lifetime values of the model component $\left(\mathrm{E}\left(\tau_{i}\right)\right)$ create strictly monotonic series corresponding to the requirement by Equation (32) (Table 8).

The comparison of the mechanical behavior of the two composites is performed by calculating the components and the resultant of the reliability functions (Figure 16) and weighted resistant forces (Figure 17). The vertical dotted black lines in Figures 16 and 17 indicate the peak value of the measured tensile force $\left(t^{*}\right)$. In the composite sample without a coupling agent (Figure 16.a), at lower loads the matrix damages (D11) and debonding (D12) determine the damage process, then the reliability, which is the number of the intact elements (model fibers), quickly decreases while at higher load the slippage (D13) and breakage (D14) of fibers dominate before ultimate failure. As opposed to that, in the case of the composite treated with a coupling agent (Figure 16.b), fiber-based damage (D23-D25) governs the whole deformation and damage process producing far higher reliability.

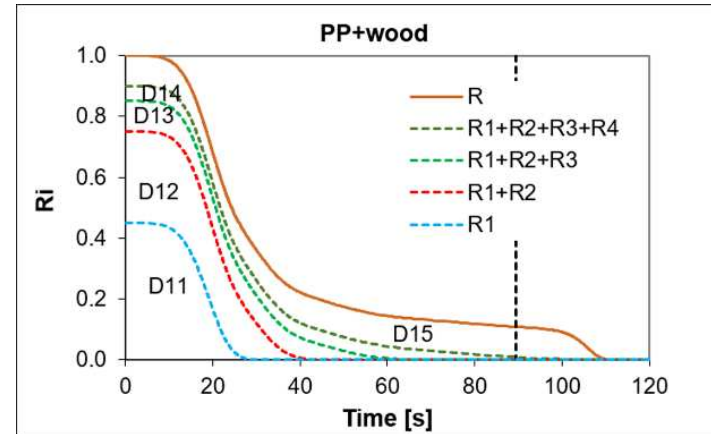

(a)

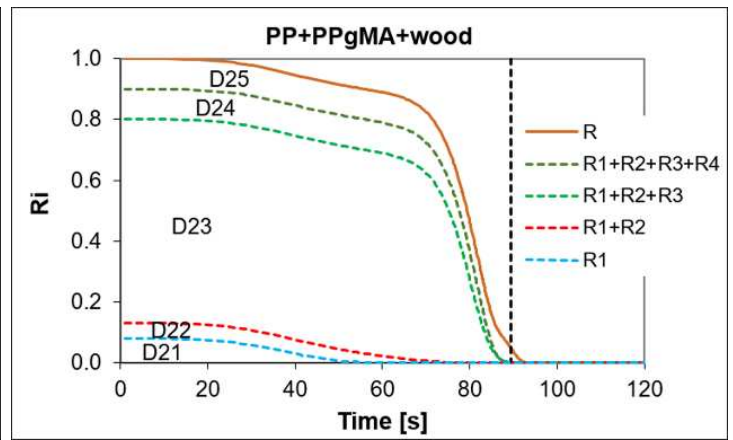

(b)

Figure 16. The fraction of different damage modes (Dji) vs. the time of PP/WF composites without $(\mathrm{a} ; j=1)$ and with $(\mathrm{b} ; j=2)$ a coupling agent (PPgMA)

Figure 17 shows the variation of the component fraction of the $\mathrm{AE}$ event numbers as a function of load time according to Equation (43), confirming the statements above.

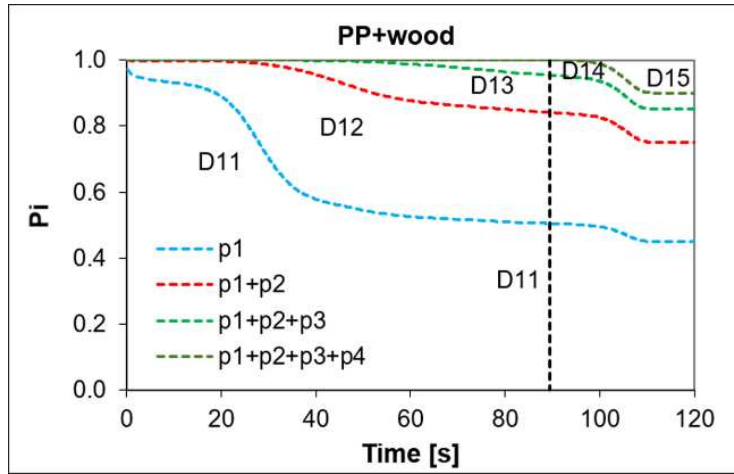

(a)

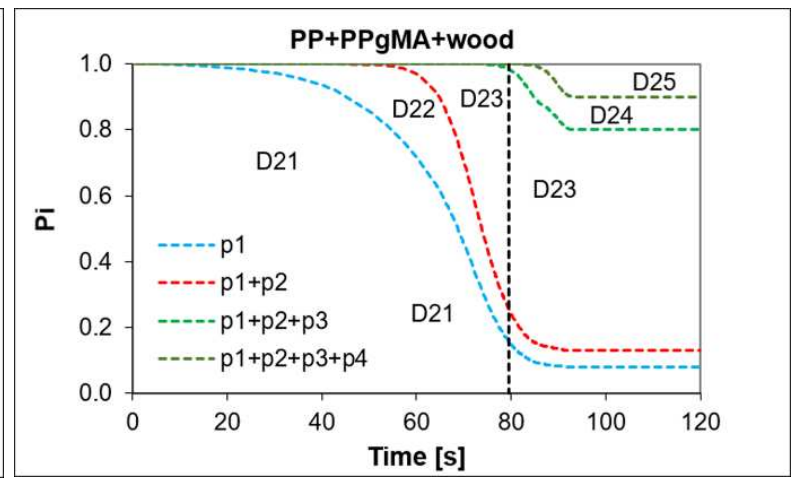

(b)

Figure 17. The fraction of different damage modes (Dji) vs. the time of PP/WF composites without $(\mathrm{a} ; j=1)$ and with $(\mathrm{b} ; j=2)$ a coupling agent (PPgMA) 
The component fraction of the AE event number can also show in itself the difference in the mechanical behavior of the two composites since, as Figure 17 indicates, in the case of the composite without a coupling agent (Figure 17.a), there are four significant damage modes (D11-D14) before the force peak, while the use of this agent (Figure 17.b) decreases the number to three (D21-D23), although in the latter case the third damage mode (D23) dominates the AE event process around the force peak.

In both cases, the fifth component (D15, D25) modeling the ultimate failure of the remaining intact part of the sample plays a virtual role appearing beyond the force peak only.

\section{Conclusions}

In general, the main objective of $\mathrm{AE}$ assisted mechanical tests is to identify and determine the strength properties of the different failure modes characterizing the material and the loading mode.

The presented fiber-bundle-cells (FBC) based modeling method can evaluate a simultaneous tensile test and $\mathrm{AE}$ measurements. It makes it possible to decompose the measured tensile force and $\mathrm{AE}$ event number processes and to determine the number fraction of the components obtained and the distributions of their strength, that is, the load levels where the micro-failures occur, and the amplitude and energy of the AE signals generated by the microfailures. In addition, the number fraction of the AE events generated by different failure modes can be calculated as a function of the load time, providing a failure map characterizing the mechanical behavior and the load bearing ability of the material tested.

All this was demonstrated by the compact tension (CT) testing of short fiber reinforced VERTON PP sheets as well as injection molded wood fiber reinforced PP composites, without and with a coupling agent and the results proved the applicability of the FBC modeling and evaluation method proposed. The reliability functions and the failure maps seem to give important and comprehensive information on the mechanical behavior and the damage process of the samples subjected to tensile load.

Evaluation can be improved with the use of multivariate statistical methods, such as cluster analysis, to assess the number of significant damage modes without performing structural investigation on the samples at different tensile loads or after fracture.

\section{Acknowledgements}

This work was supported by the Hungarian National Research, Development and Innovation Office (NKFIH) through grants OTKA K116189, K116070, and K120592. It was connected to the projects BME-Nanotechnology FIKP grant of EMMI (BME FIKP-NANO) and the 
NVKP_16-1-2016-0046 grant of The National Research, Development and Innovation Office (NRDIO). 


\section{References}

1. Dahmene F, Yaacoubi S and El Mountassir M. Acoustic emission of composites structures: Story, success, and challenges. In: Declercq NF (ed) Proceedings of the 2015 ICU International Congress on Ultrasonics. Amsterdam: Elsevier Science Bv, 2015, pp.599-603.

2. Sikorski W. Acoustic emission. InTech, Rijeka, 2012.

3. Ziehl P and Pollock A. Acoustic emission for civil structures. In: Sikorski W (ed) Acoustic Emission. Rijeka: InTech, 2012.

4. Romhany G, Czigany T and Karger-Kocsis J. Failure assessment and evaluation of damage development and crack growth in polymer composites via localization of acoustic emission events: A Review. Polym Rev 2017; 57: 397-439.

5. Corsini A and Sheard AG. End-plate for noise-by-flow control in axial fans. Periodica Polytechnica Mechanical Engineering 2013; 57: 3-6.

6. Kalmár-Nagy T, Bak BD, Benedek T, et al. Vibration and noise of an axial flow fan. Periodica Polytechnica Mechanical Engineering 2015; 59: 109-113.

7. Shippen NC and Adams DF. Acoustic emission monitoring of damage progression in graphite/epoxy laminates. J Reinf Plast Compos 1985; 4: 242-261.

8. Faudree $\mathrm{M}, \mathrm{Baer} \mathrm{E}$, Hiltner $\mathrm{A}$, et al. Characterization of damage and fracture processes in short fiber BMC composites by acoustic-emission. J Compos Mater 1988; 22: 1170-1195.

9. Hill R and Okoroafor EU. Weibull statistics of fiber bundle failure using mechanical and acoustic-emission testing - The influence of interfiber friction. Composites 1995; 26: 699-705.

10. Czigany $T$ and Karger-Kocsis J. Comparison of the failure mode in short and long glass-fiberreinforced injection-molded polypropylene composites by acoustic-emission. Polym Bull 1993; 31: 495-501.

11. Luo JJ, Wooh SC and Daniel IM. Acoustic-emission study of failure mechanisms in ceramicmatrix composite under longitudinal tensile loading. J Compos Mater 1995; 29: 1946-1961.

12. Shiwa $\mathrm{M}$, Carpenter $\mathrm{S}$ and Kishi T. Analysis of acoustic emission signals generated during the fatigue testing of GFRP. J Compos Mater 1996; 30: 2019-2041.

13. Velu YK, Ellison MS, Dai JR, et al. Single fiber acoustic emission and tensile properties from cotton bundle fracture tests. Text Res J 1999; 69: 616-620.

14. Czigány $\mathrm{T}$. The role of the acoustic emission in the fracture-mechanical examination of plastic composites (in Hungarian). Anyagvizsgálók Lapja 1998; 8: 13-16.

15. Zhuang XM and Yan X. Investigation of damage mechanisms in self-reinforced polyethylene composites by acoustic emission. Compos Sci Technol 2006; 66: 444-449.

16. Oskouei AR and Ahmadi M. Fracture strength distribution in E-glass fiber using acoustic emission. J Compos Mater 2010; 44: 693-705.

17. Fotouhi M, Pashmforoush F, Ahmadi M, et al. Monitoring the initiation and growth of delamination in composite materials using acoustic emission under quasi-static three-point bending test. J Reinf Plast Compos 2011; 30: 1481-1493.

18. Santulli C, Kuan HT, Sarasini F, et al. Damage characterisation on PP-hemp/aluminium fibremetal laminates using acoustic emission. J Compos Mater 2013; 47: 2265-2274.

19. Awerbuch J, Leone FA, Jr., Ozevin D, et al. On the applicability of acoustic emission to identify modes of damage in full-scale composite fuselage structures. J Compos Mater 2016; 50: 447-469.

20. Hari J, Horvath F, Moczo J, et al. Competitive interactions, structure and properties in polymer/layered silicate nanocomposites. Express Polym Lett 2017; 11: 479-492.

21. de Groot PJ, Wijnen PAM and Janssen RBF. Real-time frequency determination of acoustic emission for different fracture mechanisms in carbon epoxy composites. Compos Sci Technol 1995; 55: 405-412.

22. Giordano M, Calabro A, Esposito $C$, et al. An acoustic-emission characterization of the failure modes in polymer-composite materials. Compos Sci Technol 1998; 58: 1923-1928. 
23. Bohse J. Acoustic emission characteristics of micro-failure processes in polymer blends and composites. Compos Sci Technol 2000; 60: 1213-1226.

24. Fotouhi M and Najafabadi MA. Acoustic emission-based study to characterize the initiation of delamination in composite materials. J Thermoplast Compos Mater 2016; 29: 519-537.

25. Lomov SV, Karahan M, Bogdanovich AE, et al. Monitoring of acoustic emission damage during tensile loading of 3D woven carbon/epoxy composites. Text Res J 2014; 84: 1373-1384.

26. Bak KM, Kalaichelvan K, Jothilingam A, et al. Acoustic emission characterization of failure modes of single-lap joints in glass/epoxy specimens. J Compos Mater 2016; 50: 3-23.

27. Ni QQ and Iwamoto M. Wavelet transform of acoustic emission signals in failure of model composites. Eng Fract Mech 2002; 69: 717-728.

28. Kamala G, Hashemi J and Barhorst AA. Discrete-wavelet analysis of acoustic emissions during fatigue loading of carbon fiber reinforced composites. J Reinf Plast Compos 2001; 20: 222-238.

29. Oskouei AR, Ahmadi M and Hajikhani M. Wavelet-based acoustic emission characterization of damage mechanism in composite materials under mode I delamination at different interfaces.

Express Polym Lett 2009; 3: 804-813.

30. Lu C, Ding P and Chen ZH. Time-frequency Analysis of Acoustic Emission Signals Generated by Tension Damage in CFRP. Procedia Eng 2011; 23: 210-215.

31. Heidary $\mathrm{H}$, Ahmadi $\mathrm{M}$, Rahimi A, et al. Wavelet-based acoustic emission characterization of residual strength of drilled composite materials. J Compos Mater 2013; 47: 2897-2908.

32. Bak KM, KalaiChelvan K, Vijayaraghavan GK, et al. Acoustic emission wavelet transform on adhesively bonded single-lap joints of composite laminate during tensile test. J Reinf Plast Compos 2013; 32: 87-95.

33. Hafizi ZM, Epaarachchi J and Lau KT. An investigation of acoustic emission signal attenuation for monitoring of progressive failure in fiberglass reinforced composite laminates. International Journal of Automotive and Mechanical Engineering 2013; 8: 1442-1456.

34. $\mathrm{Wu} Q, \mathrm{Yu} F, \mathrm{Okabe} \mathrm{Y}$, et al. Acoustic emission detection and position identification of transverse cracks in carbon fiber-reinforced plastic laminates by using a novel optical fiber ultrasonic sensing system. Struct Health Monit 2015; 14: 205-213.

35. Wu G, Zhong $\mathrm{Y}$ and $\mathrm{Yu} \mathrm{W}$. Identifying the breaks of wool fibers based on the waveform analysis of acoustic emission. Text Res J 2015; 85: 1319-1330.

36. Fotouhi M, Saeedifar M, Sadeghi S, et al. Investigation of the damage mechanisms for mode I delamination growth in foam core sandwich composites using acoustic emission. Struct Health Monit 2015; 14: 265-280.

37. Bocchieri RT, Schapery RA and Gorman MR. Time-dependent microcracking detected in a rubber-toughened carbon-epoxy composite by the modal acoustic emission method. I Compos Mater 2003; 37: 421-451.

38. Burks B and Kumosa M. A modal acoustic emission signal classification scheme derived from finite element simulation. Int J Damage Mech 2014; 23: 43-62.

39. Karger-Kocsis J and Fejes-Kozma Z. Failure mode and damage zone development in a GMT-PP by acoustic-emission and thermography. J Reinf Plast Compos 1994; 13: 768-792.

40. Fu T, Zhang Z, Liu Y, et al. Development of an artificial neural network for source localization using a fiber optic acoustic emission sensor array. Struct Health Monit 2015; 14: 168-177.

41. Okafor AC, Singh N, Singh N, et al. Acoustic emission detection and prediction of fatigue crack propagation in composite patch repairs using neural network. J Thermoplast Compos Mater 2015; 30: 3-29.

42. Castellano A, Fraddosio A and Piccioni MD. Quantitative analysis of QSI and LVI damage in GFRP unidirectional composite laminates by a new ultrasonic approach. Compos Part B-Eng 2018; 151: 106-117.

43. Rakotonarivo ST, Payan C, Moysan J, et al. Local damage evaluation of a laminate composite plate using ultrasonic birefringence of shear wave. Compos Part B-Eng 2018; 142: 287-292.

44. Wronkowicz A, Dragan K and Lis K. Assessment of uncertainty in damage evaluation by ultrasonic testing of composite structures. Compos Struct 2018; 203: 71-84. 
45. Manson $\mathrm{G}$, Worden $\mathrm{K}$, Holford K, et al. Visualisation and dimension reduction of acoustic emission data for damage detection. J Intell Mater Syst Struct 2001; 12: 529-536.

46. Ativitavas N, Pothisiri T and Fowler TJ. Identification of fiber-reinforced plastic failure mechanisms from acoustic emission data using neural networks. J Compos Mater 2006; 40: 193-226.

47. Pashmforoush F, Fotouhi M and Ahmadi M. Acoustic emission-based damage classification of glass/polyester composites using harmony search k-means algorithm. J Reinf Plast Compos 2012; 31: 671-680.

48. Wang X, Gao X-Z and Zenger K. The Overview of Harmony Search. An Introduction to Harmony Search Optimization Method. Cham: Springer International Publishing, 2015, pp.5-11. 49. Silversides I, Maslouhi A and LaPlante G. Acoustic emission monitoring of interlaminar delamination onset in carbon fibre composites. Struct Health Monit 2013; 12: 126-140.

50. Assarar M, Bentahar M, El Mahi A, et al. Monitoring of damage mechanisms in sandwich composite materials using acoustic emission. Int J Damage Mech 2015; 24: 787-804.

51. Al-Jumaili SK, Holford KM, Eaton MJ, et al. Classification of acoustic emission data from buckling test of carbon fibre panel using unsupervised clustering techniques. Struct Health Monit 2015; 14: 241-251.

52. Heidary $\mathrm{H}$, Karimi NZ, Ahmadi $\mathrm{M}$, et al. Clustering of acoustic emission signals collected during drilling process of composite materials using unsupervised classifiers. J Compos Mater 2015; 49: 559-571.

53. McCrory JP, Al-Jumaili SK, Crivelli D, et al. Damage classification in carbon fibre composites using acoustic emission: A comparison of three techniques. Compos Part B-Eng 2015; 68: 424-430.

54. Belchamber RM, Betteridge D, Chow YT, et al. A study of acoustic emissions from stressed polypropylene - glass-fiber composites. J Compos Mater 1983; 17: 420-434.

55. Vas LM and Racz Z. Modeling and testing the fracture process of impregnated carbon-fiber roving specimens during bending: Part I - Fiber bundle model. J Compos Mater 2004; 38: 1757-1785.

56. Vas LM, Racz $Z$ and Nagy P. Modeling and testing the fracture process of impregnated carbon-fiber roving specimens during bending: Part II - Experimental studies studies. J Compos Mater 2004; 38: 1787-1801.

57. Vas LM. Strength of unidirectional short fiber structures as a function of fiber length. $J$ Compos Mater 2006; 40: 1695-1734.

58. Vas LM. Statistical fiber-bundle-cells and their application to modeling fibrous structures, composites (in Hungarian). DSc Thesis, Hungarian Academy of Sciences, Budapest, 2007.

59. Vas LM and Tamas P. Modelling method based on idealised fibre bundles. Plast Rubber Compos 2008; 37: 233-239.

60. Kocsis Z. Development of wood fiber reinforced composites PhD Thesis, Budapest University of Technology and Economics, Budapest, 2008.

61. Molnar K, Vas LM and Czigany T. Determination of tensile strength of electrospun single nanofibers through modeling tensile behavior of the nanofibrous mat. Compos Part B-Eng 2012; 43: $15-21$.

62. Bendat JS and Piersol AG. Random data: Analysis and measurement procedures. 4th ed. New York: John Wiley \& Sons, 2010.

63. Feller W. An introduction to probability theory and its applications. 3 ed. New York: John Wiley \& Sons, 1968. 